\title{
Energy-Efficient Channel-Dependent Cooperative Relaying for the Multi-User SC-FDMA Uplink
}

\author{
Jiayi Zhang, Student Member, IEEE, Lie-Liang Yang, Senior Member, IEEE, and Lajos Hanzo, Fellow, IEEE
}

\begin{abstract}
In this contribution we exploit the benefits of combining the diversity gains arising from cooperation, multiple propagation paths and from the opportunistic relaying of multiple users. Our goal is to improve the energy-efficiency of the amplify-and-forward single-relay assisted single-carrier frequency-division multiple-access uplink, where the single relay considered may support a single user or may be shared by multiple users communicating over dispersive channels subject to large-scale fading. Based on the proposed amalgam of single-tap frequency-domain equalisation and a diversity combining aided receiver relying on the minimum mean-square error criterion, three different relay selection schemes designed for either singleuser or multi-user relaying scenarios are investigated, when combined with source/relay power sharing, which employ imperfect power control. Our results demonstrate that at a bit error ratio of $10^{-4}$, the proposed receiver is capable of saving $2 \mathrm{~dB}$ power by achieving a higher cooperative diversity gain than the conventional receiver. Moreover, a beneficial energy-efficiency improvement may be achieved, when the cooperative regime operates at $E_{b} / N_{0}<0$. Most importantly, when the shadowing variance is increased from $4 \mathrm{~dB}$ to $8 \mathrm{~dB}$, the energy consumption gain gleaned from our multi-user and multi-access relay selection schemes may increase to $4 \sim 9$ compared to the direct transmission in the absence of shadowing at $E_{b} / N_{0}=-10 \mathrm{~dB}$.
\end{abstract}

Index Terms-Cooperative Communications, Diversity Combining, Energy-Efficiency, Frequency-Domain Equlisation, Opportunistic Relaying, Power Allocation, SC-FDMA, Relay Selection

\section{INTRODUCTION}

C OOPERATIVE COMMUNICATION [1] systems have attracted the attention of both academia and industry in recent years, since they are capable of achieving a diversity gain in large-scale fading environments by sharing the resources of the cooperating user terminals. This allows us to jointly exploit the benefits of both time- and frequencydiversity for the sake of mitigating the deleterious effects of wireless propagation and/or for increasing the attainable system throughput and energy-efficiency [2]-[5]. Recently, the family of cooperative diversity oriented Multiple-Access (MA) and distributed Multiple-Input Multiple-Output (MIMO)

Copyright (C2011 IEEE. Personal use of this material is permitted. However, permission to use this material for any other purposes must be obtained from the IEEE by sending a request to pubs-permissions@ieee.org.

The authors are with the School of Electronics and Computer Science, University of Southampton, SO17 1BJ, UK. Email: \{jz07r, 1ly, lh\}@ecs.soton.ac.uk; http://www-mobile.ecs.soton.ac.uk.

The work reported in this paper has formed part of the Core 5 Research Programme of the Virtual Centre of Excellence in Mobile and Personal Communications, Mobile VCE, www.mobilevce.com, whose funding support, including that of EPSRC, is gratefully acknowledged. Fully detailed technical reports on this research are available to Industrial Members of Mobile VCE.

This work was presented in part at IEEE VTC2010-Spring, 16-19 May 2010, Taipei.

Manuscript received 12 April, 2010; revised 24 July, 2010, 18 Oct. 2010 and 20 Dec. 2010; accepted 30 Dec. 2010. aided multiplexing techniques has been invoked in order to design the uplink of advanced cooperative cellular networks [6]-[8]. Furthermore, the cooperative concepts have been extended to broadband systems by designing techniques for mitigating the effects of frequency-selective fading with the aid of Multi-Carrier (MC) techniques associated with appropriate source/relay power sharing [9], [10].

From a Multi-User (MU) network point of view, the cooperative link sharing from the source Mobile Terminals (MT) to the Base Station (BS) can be determined by choosing the single or multiple relays [11] from a cluster of idle MTs. Generally, the Random Relay Selection (RRS) philosophy allows the BS to appoint a relay randomly without any channel knowledge, but in this case simultaneous gains from relaying path and selection diversity are limited. By contrast, the socalled Distance-Dependent Relay Selection (DD-RS) [8] policy is based on the distance from the relay to the source MT or BS, hence the Relay Candidates (RCs) which benefits from a high path gain may experience deep shadowing and fast fading. However, the Channel-Dependent Relay Selection (CD-RS) regime benefits from a certain degree-of-freedom in terms of selecting the cooperating MT, by monitoring the instantaneous channel conditions in a distributed scenario, including the associated path-loss, shadowing and multi-path fading effects. Therefore, it is also known as Opportunistic Relaying (OR) [12], which is capable of exploiting the selection diversity that we refer to as multi-user diversity arising from appropriate relay selection [13]-[16].

The Single-Carrier Frequency-Domain Multiple-Access (SC-FDMA) technique [17] was adopted for the uplink of the 3-rd Generation Partnership Project's (3GPP) Long Term Evolution (LTE) standard [18]. In recent years Falconer et al. [19], [20] investigated the SC modulation with linear and nonlinear Frequency-Domain Equalisation (FDE) [21]techniques for broadband receiver solutions. Furthermore, the principle of SC-FDMA and its Discrete Fourier Transform (DFT) spread Orthogonal Frequency-Division Multiplexing (OFDM) [22] transmitter structures using either interleaved or localised subband mapping schemes were studied in [23], [24]. SCFDMA was shown to be capable of avoiding the Multi-User Interference (MUI) imposed by the cooperative sources and relays upon the uplink receiver of the BS, while maintaining a low Peak-to-Average Power Ratio (PAPR). The Amplifyand-Forward (AF) single-relay assisted SC-FDMA uplink scheme was proposed in [25] for both Single-DedicatedRelaying (SDR) with each dedicated relay aiding a single user and Single-Shared-Relaying (SSR) with a single shared relay aiding all the active users. The AF relay estimates the received power of each subband and equalises the powerdifferences of the subbands, which corresponds to subband- 
based equalisation. By inheriting the features of the SCFDMA system invoking a DFT-S-OFDM style transmitter, this relaying scheme carries out so-called subband remapping [25] at the relay in order to remove the effects of both noise and interference inflicted by other relays without changing the frequency band of the signals transmitted from the source MT. An OFDM scheme relying on time-division multiplexing based cooperative relaying and using Minimum Mean-Square Error (MMSE) assisted frequency-domain equalisers (FDE) was proposed in [26]. However, in [25], [26] the MMSE-FDE was operated independently in the context of the direct and relaying branches, which were then combined with the aid of a Time-Domain (TD) Equal-Gain Combiner (EGC) [27]. In [28]-[32], linear and adaptive FDEs relying on diversity combining schemes were invoked for multiple antenna aided SCor OFDM-based block transmissions. Furthermore, in [33] [35] the receive diversity combining techniques designed for SC-FDMA and relying on cooperative relays were considered by stipulating the idealised simplifying assumption that the relays demodulate/detect the source signals perfectly, before forwarding them.

- Although the authors of the above-mentioned articles proposed various FDEs amalgamated with diversity combining schemes designed for MIMOs, for OFDM or for relaying systems, owing to the potentially non-white noise contribution of AF cooperative relaying, the equivalent noise encountered at the BS is also non-white. Furthermore, we typically encounter a different noise power at each cooperative branch, hence the conventional maximum ratio combiner (MRC) [27] becomes suboptimal, unless noise whitening is adopted. To the best of our knowledge, no article investigated the joint design of the linear single-tap FDE amalgamated with the diversity combiner of the direct- and relayed-link using noisewhitening for the Amplify-and-Forward (AF) relaying assisted SC-FDMA uplink.

- Since finite-delay power control schemes using a discrete stepsize cannot perform perfectly in realistic wireless uplink transmissions, the source/relay power sharing employed imposes a time-varying level of Power Control Error (PCE) [36]. In [6], [9], [10], [13], various relay selection and source/relay power allocation schemes were proposed and studied, but no such schemes were designed for the cooperative SC-FDMA uplink under imperfect power control with the goal of improving the energyefficiency.

- The authors of [37]-[39] studied various relay selection schemes designed for OFDM and CDMA systems subjected to both path-loss and multi-path fading. However, cooperative relaying allows the collaborating mobiles to avoid the typical diversity gain erosion imposed by shadowing effects, as a benefit of their geographically separated locations. Hence we embarked on investigating the relay selection induced benefits of our system in the presence of shadow fading, whose impact on the energyefficiency of cooperative SC-FDMA systems has not been documented in the open literature.
In this paper, we propose and investigate an energyefficient opportunistic cooperative relaying scheme designed for SC-FDMA arrangements. The Joint Frequency-Domain Equalisation and Combining (JFDEC) aided receiver is designed to detect the cooperative SC-FDMA uplink signals. In this paper, both Single-User Relay Selection (SU-RS) and Multi-User Relay Selection (MU-RS) as well as MultipleAccess Relay Selection (MA-RS) are considered in diverse opportunistic cooperative relaying scenarios. By contrast, in [40] only the SU-RS and MU-RS schemes based on the JFDEC receiver were studied in the context of the SDR topology.

- Furthermore, in contrast to the open literature, our focus is mainly on the analysis of the energy-efficiency of the cooperative SC-FDMA system, where the energyefficiency is quantified in terms of the Energy Consumption Ratio (ECR) and Energy Consumption Gain (ECG), as defined in [41].

- The MMSE criterion aided JFDEC scheme considered in [40] is further detailed, which amalgamates the design of the linear single-tap FDE and that of the diversity combiner of the direct-and relayed-link with noise whitening applied at the BS.

- Furthermore, we investigate the MA-RS scheme created by generalising the $S U-R S$ and $M U-R S$ philosophies developed from the context of the SDR to SSR topologies, where the relative merits of the $S U-R S, M U-R S$ and MA$R S$ schemes are discussed in the light of their complexity and the effects of imperfect power control ${ }^{1}$ are also quantified.

- Additionally, we demonstrate that upon encountering a realistic propagation path-loss and shadowing, a significant relaying gain and selective diversity gain are attainable by the proposed Optimal Power Allocation $(O P A)^{2}$ aided $S U-R S, M U-R S$ and MA-RS schemes, which allows us to reduce the required Signal-to-Interferenceplus-Noise Ratio (SINR) for the sake of improving the performance of ECG. Finally, the practicability and feasibility of various power sharing schemes are discussed in terms of their computational complexity.

This treatise is organised as follows. In Section II, the system model of the cooperative SC-FDMA uplink is presented. In Sections III and IV, we outline the improved signal detection and investigate the relay selection schemes, respectively. The attainable performance of our proposed schemes is quantified by the simulation results of Section V. Finally, we conclude in Section VI.

\section{RELAY AsSisted SC-FDMA System MODEL}

\section{A. Transmitted Signal of Source MT}

The relay-assisted SC-FDMA system considered supports $K$ uplink users referred to as the source MTs in a cell. There are also idle terminals, which can be activated as the relays. The transmitter's block diagram is seen in the upper illustration

\footnotetext{
${ }^{1}$ Power control errors are imposed on the MT's transmit power due to the feedback delay and estimation errors at the BS.

${ }^{2}$ Optimised transmit power sharing of cooperative MTs is assigned by the BS via feedback channel by ignoring power control error.
} 


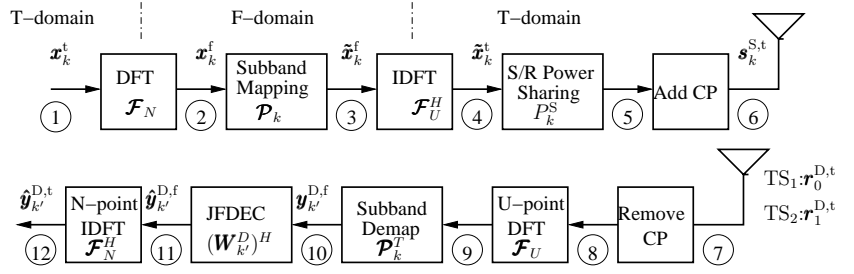

Fig. 1. DFT-spread-OFDMA style transmitter and MMSE assisted JFDEC BS receiver

of Fig. 1. The $U$-symbol baseband equivalent discrete-time signal transmitted by the $k$-th source MT before inserting the cyclic-prefix (CP) may be expressed as [25].

$$
\boldsymbol{s}_{k}^{\mathrm{S}, \mathrm{t}}=\sqrt{P_{k}^{\mathrm{S}}} \mathcal{F}_{U}^{H} \boldsymbol{\mathcal { P }}_{k} \mathcal{F}_{N} \boldsymbol{x}_{k}^{\mathrm{t}}
$$

where the superscript ${ }^{\mathrm{t}}$ refers to the TD signal, while $\mathcal{F}_{U}$ and $\mathcal{F}_{N}$ denote the normalised $U$-point and $N$-point Fast Fourier Transform (FFT) matrices, respectively. Furthermore, $\mathcal{P}_{k}$ represents the mapping of the $k$-th users symbols to the most appropriate $N$ subbands selected from the entire set of $U=M \times N$ subbands, where $M$ is the bandwidth expansion factor. We refer to this operation as subband mapping. In the FDMA context considered this subband mapping regime guarantees that the maximum number of orthogonal users supported is equal to the BW expansion factor, i.e. we have $K \leqslant M$, and the multi-user system operates at its full-load, when we have $K=M$. Moreover, the interleaved subband mapping mode in our system is defined as $\mathcal{P}_{k,(u, i)}=1$ if $u=i M+k$, otherwise $\mathcal{P}_{k,(u, i)}=0,(0<u<U-1,0<i<$ $N-1)$, where $\mathcal{P}_{k,(u, n)}$ is the $(u, n)$-th entry of $\boldsymbol{P}_{k}$ and we have $u=0,1, \cdots, U-1, n=0,1, \cdots, N-1$. Additionally, $\boldsymbol{x}_{k}^{\mathrm{t}}$ denotes the original $N$-symbol information packet of the $k$-th user. Finally, $P_{k}^{\mathrm{S}}$ is the source MT's transmitted power determined by the power allocation and subjected to a power control error as it will be discussed in Subsection II-B.

\section{B. Channel Modelling and Assumptions}

For the sake of simplicity, we assume that the source MTs, relays and $\mathrm{BS}$ are all located in a line, and the relays roam between the source and destination. Let us assume that the length of the Source-Destination (S-D) link is the reference distance in the propagation model having a path-loss exponent $\eta$ [27]. Then, the instantaneous path-loss values of the relay channels, denoted by $G_{\mathrm{SR}}$ for the Source-Relay (S-R) and $G_{\mathrm{RD}}$ for the Relay-Destination (R-D), respectively, become the corresponding relaying gains, which incorporate the effects of the average path-loss combined with shadowing. Specifically, the average path-losses of the S-R and R-D links are denoted by $\delta_{\mathrm{SR}}^{-\eta}$ and $\delta_{\mathrm{RD}}^{-\eta}$, respectively, where $\delta_{\mathrm{SR}}+\delta_{\mathrm{RD}}=1$, and $\delta_{\mathrm{SR}}$ and $\delta_{\mathrm{RD}}$ are the distance normalised by the S-D distance. The shadowing component is characterised by the log-normal distribution associated with a zero mean and a standard deviation of $\sigma_{\xi}$, i.e. we have $\xi(d B) \sim \mathcal{N}\left(0, \sigma_{\xi}^{2}\right)$. Therefore, we can write $G_{\mathrm{SR}}=\xi_{\mathrm{SR}} \delta_{\mathrm{SR}}^{-\eta}, G_{\mathrm{RD}}=\xi_{\mathrm{RD}} \delta_{\mathrm{RD}}^{-\eta}$, while the shadowing effect at the S-D link is denoted by $\xi_{\mathrm{SD}}$ [42].

Note that in a small-scale scenario, we assume that the systems are experiencing frequency-selective fading associated with $L$ paths. However, each subcarrier is assumed to experience flat fading. We assume that perfect Channel State Information (CSI) is available for both the relays and BS, including the path-loss, shadowing and fast fading.

Furthermore, in order to guarantee a fair comparison between the cooperative and non-cooperative systems, the total signal power $P$ of each user is normalised to unity. Specifically, the source and relay MTs' transmit power assigned to the $k$-th user are quantified as $P_{k}^{\mathrm{S}}=\epsilon_{k}^{\mathrm{S}} \alpha_{k}^{\mathrm{S}} P$ and $P_{k}^{\mathrm{R}}=\epsilon_{k}^{\mathrm{R}} \alpha_{k}^{\mathrm{R}} P$, respectively. The imperfect power control effects imposed on the transmitted power of the MTs can be evaluated by modelling it using the classic log-normal distributed power control error having a standard deviation of $\sigma_{\epsilon}$ in $\mathrm{dB}$, i.e. $\epsilon(d B) \sim \mathcal{N}\left(0, \sigma_{\epsilon}^{2}\right)$ [36]. Additionally, we quantify the power constraints of the source and relay as

$$
\alpha_{k}^{\mathrm{S}}+\alpha_{k}^{\mathrm{R}}=1,
$$

where $\alpha_{k}^{\mathrm{S}}$ and $\alpha_{k}^{\mathrm{R}}$ represent the power sharing between the source and relay. Note that, in the SSR topology the relay simultaneously transmits all the $K$ users' signals, while obeying the power constraint given by

$$
\alpha_{\mathrm{R}}=\sum_{k=0}^{K-1} \alpha_{k}^{\mathrm{R}}=1 .
$$

\section{Relaying Models}

In order to separate multiple users in the FD and hence to avoid the MUI, each source MT is assigned a single relay by the $\mathrm{BS}$ according to the opportunistic relaying mechanism to be detailed in our forthcoming discourse in Section IV. The block diagram of AF relay model is shown in Fig. 2. We assume that the cooperation is half-duplex time-division based. Hence during the first time-slot $\left(\mathrm{TS}_{1}\right)$ all the $K$ source MTs broadcast their messages represented by $\boldsymbol{s}_{k}^{\mathrm{S}, \mathrm{t}},(k=0,1, \cdots, K-1)$, which are received by both the relays and the $\mathrm{BS}$ via the $\mathrm{S}-\mathrm{R}$ and $\mathrm{S}-\mathrm{D}$ links, respectively. During the second time-slot $\left(\mathrm{TS}_{2}\right)$, which is the cooperationphase, we consider both the SDR and SSR scenarios.

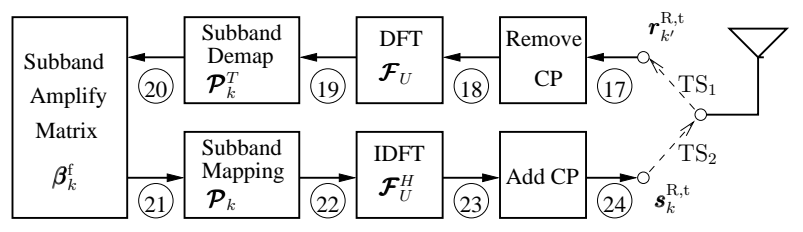

Fig. 2. AF relay structure

In the SDR system, the relays only forward the signals from the dedicated source MT to the BS via the R-D links, implying that a total of $K$ relays are required for $K$ source MTs. The signal received by the $k^{\prime}$-th relay $\left(k^{\prime}=0,1, \cdots, K-1\right)$ as well as by the $\mathrm{BS}$ during $\mathrm{TS}_{1}$ and $\mathrm{TS}_{2}$, respectively, are expressed by the $U$-length vectors of

$$
\begin{aligned}
& \boldsymbol{r}_{k^{\prime}}^{\mathrm{R}, \mathrm{t}}=\sqrt{G_{\mathrm{SR}}} \sum_{k=0}^{K-1} \tilde{\boldsymbol{H}}_{k}^{\mathrm{SR}, \mathrm{t}} \boldsymbol{s}_{k}^{\mathrm{S}, \mathrm{t}}+\tilde{\boldsymbol{n}}_{k}^{\mathrm{R}, \mathrm{t}}, \\
& \boldsymbol{r}_{1}^{\mathrm{D}, \mathrm{t}}=\sqrt{G_{\mathrm{RD}}} \sum_{k^{\prime}=0}^{K-1} \tilde{\boldsymbol{H}}_{k^{\prime}}^{\mathrm{RD}, \mathrm{t}} \boldsymbol{s}_{k^{\prime}}^{\mathrm{R}, \mathrm{t}}+\tilde{\boldsymbol{n}}_{1}^{\mathrm{D}, \mathrm{t}},
\end{aligned}
$$


where $\tilde{\boldsymbol{H}}_{k}^{\mathrm{SR}, \mathrm{t}}$ and $\tilde{\boldsymbol{H}}_{k^{\prime}}^{\mathrm{RD}, \mathrm{t}}$ host the $(U \times U)$ TD channel coefficient matrices of the S-R and R-D links for the $k$-th and $k^{\prime}$-th source MT's signals, respectively, while $\tilde{\boldsymbol{n}}_{k}^{\mathrm{R}, \mathrm{t}}$ and $\tilde{\boldsymbol{n}}_{1}^{\mathrm{D}, \mathrm{t}}$ represent the $U$ length complex-valued AWGN vectors having a zero mean and a variance of $\sigma_{N}^{2}$ at each element, i.e. we have $\mathcal{C N}\left(0, \sigma_{N}^{2}\right)$ at both the $k$-th relay and the $\mathrm{BS}$, respectively.

Alternatively, a single relay may be shared by $K$ source MTs to form a SSR uplink. Therefore, the representation of the signals received at the relay and BS is given by

$$
\begin{aligned}
& \boldsymbol{r}^{\mathrm{R}, \mathrm{t}}=\sqrt{G_{\mathrm{SR}}} \sum_{k=0}^{K-1} \tilde{\boldsymbol{H}}_{k}^{\mathrm{SR}, \mathrm{t}} \boldsymbol{s}_{k}^{\mathrm{S}, \mathrm{t}}+\tilde{\boldsymbol{n}}_{\mathrm{R}, \mathrm{t}} \\
& \boldsymbol{r}_{1}^{\mathrm{D}, \mathrm{t}}=\sqrt{G_{\mathrm{RD}}} \tilde{\boldsymbol{H}}^{\mathrm{RD}, \mathrm{t}} \boldsymbol{s}^{\mathrm{R}, \mathrm{t}}+\tilde{\boldsymbol{n}}_{1}^{\mathrm{D}, \mathrm{t}},
\end{aligned}
$$

where all the $K$ source signals are embedded in the forwarded messages, which is denoted by $s^{\mathrm{R}, \mathrm{t}}$.

By invoking the subband-based equalisation aided AF scheme, the relay's received TD signals are firstly transformed to the FD by the $U$-point DFT operation and then demapped to the appropriate $N$ subbands by $\mathcal{P}_{k}^{T}$, which we refer to as subband demapping [24]. Each user's resultant signal is multipled by the $(N \times N)$-element diagonal matrix

$$
\boldsymbol{\beta}_{k}^{\mathrm{f}}=\operatorname{diag}\left\{\beta_{k, 0}^{\mathrm{f}}, \beta_{k, 1}^{\mathrm{f}}, \cdots, \beta_{k,(N-1)}^{\mathrm{f}}\right\},
$$

where the $n$-th element is the specific gain factor of the $n$-th subband, yielding [25]

$$
\left.\beta_{k, n}^{\mathrm{f}}=\sqrt{P_{k}^{\mathrm{R}} /\left[P_{k}^{\mathrm{S}} G_{\mathrm{SR}}\left|h_{k, n}^{\mathrm{SR}, \mathrm{f}}\right|^{2}+\sigma_{N}^{2}\right.}\right] .
$$

Then the relay's signal corresponding to the $k$-th user is mapped to the subbands assigned to the $k$-th source MT. We refer to the joint subband mapping and demapping procedure as the subband remapping operation. Therefore, the relay's transmission is free from interference, since neither the source MT nor the relay inflict interference during relaying. After that, the $U$-point IDFT operation is invoked to transform the signal to the TD, before it is transmitted to the BS. Finally, the transmitted signal of the $k$-th relay during $\mathrm{TS}_{2}$ is expressed as

$$
\boldsymbol{s}_{k}^{\mathrm{R}, \mathrm{t}}=\sqrt{P_{k}^{\mathrm{S}} G_{\mathrm{SR}}} \mathcal{F}_{U}^{H} \boldsymbol{P}_{k} \boldsymbol{\beta}_{k}^{\mathrm{f}} \boldsymbol{H}_{k}^{\mathrm{SR}, \mathrm{f}} \boldsymbol{x}_{k}^{\mathrm{f}}+\overline{\boldsymbol{n}}_{k}^{\mathrm{R}, \mathrm{t}},
$$

where $\boldsymbol{H}_{k}^{\mathrm{SR}, \mathrm{f}}$ represents the $k^{\text {th }}$ user's $(N \times N)$-element diagonal equivalent FD channel matrix characterising the S-R link, i.e. we have

$$
\begin{aligned}
\boldsymbol{H}_{k}^{\mathrm{SR}, \mathrm{f}} & =\mathcal{P}_{k}^{T} \mathcal{F}_{U} \tilde{\boldsymbol{H}}_{k}^{\mathrm{SR}, \mathrm{t}} \mathcal{F}_{U}^{H} \mathcal{P}_{k} \\
& =\operatorname{diag}\left\{h_{k, 0}^{\mathrm{SR}, \mathrm{f}}, h_{k, 1}^{\mathrm{SR}, \mathrm{f}}, \cdots, h_{k,(N-1)}^{\mathrm{SR}, \mathrm{f}}\right\}
\end{aligned}
$$

Note that the noise imposed on the $k$-th user's signal is affected at the relay as

$$
\overline{\boldsymbol{n}}_{k}^{\mathrm{R}, \mathrm{t}}=\mathcal{F}_{U}^{H} \boldsymbol{\mathcal { P }}_{k} \boldsymbol{\beta}_{k}^{\mathrm{f}} \boldsymbol{\mathcal { P }}_{k}^{T} \mathcal{F}_{U} \tilde{\boldsymbol{n}}_{k}^{\mathrm{R}, \mathrm{t}}
$$

where the noise imposed on the other users' subbands is removed, as an additional benefit.

\section{Signal Detection}

\section{A. Representation of Received Signal at the BS}

The BS receiver structure is portrayed in the lower part of Fig. 1. After removing the CP, the $U$-point DFT transforms the TD signal to the FD, followed by subband demapping at the $\mathrm{BS}$ receiver. Then the FD signals of the $k$-th user received via the direct branch during $\mathrm{TS}_{1}$ and those arriving via the relaying branch during $\mathrm{TS}_{2}$ can be expressed by the $N$-symbol vectors of

$$
\begin{aligned}
& \boldsymbol{y}_{0, k^{\prime}}^{\mathrm{D}, \mathrm{f}}=\sqrt{P_{k^{\prime}}^{\mathrm{S}} \xi_{\mathrm{SD}}} \boldsymbol{H}_{k^{\prime}}^{\mathrm{SD}, \mathrm{f}} \boldsymbol{x}_{k^{\prime}}^{\mathrm{f}}+\boldsymbol{n}_{0}^{\mathrm{D}, \mathrm{f}} \\
& \boldsymbol{y}_{1, k^{\prime}}^{\mathrm{D}, \mathrm{f}}=\sqrt{P_{k^{\prime}}^{\mathrm{S}} G_{\mathrm{RD}} G_{\mathrm{SR}}} \boldsymbol{H}_{k^{\prime}}^{\mathrm{RD}, \mathrm{f}} \boldsymbol{\beta}_{k^{\prime}}^{\mathrm{f}} \boldsymbol{H}_{k^{\prime}}^{\mathrm{SR}, \mathrm{f}} \boldsymbol{x}_{k^{\prime}}^{\mathrm{f}}+\overline{\boldsymbol{n}}_{1}^{\mathrm{D}, \mathrm{f}},
\end{aligned}
$$

where $\boldsymbol{H}_{k^{\prime}}^{\mathrm{SD}, \mathrm{f}}$ and $\boldsymbol{H}_{k^{\prime}}^{\mathrm{RD}, \mathrm{f}}$ are the $(N \times N)$-element equivalent diagonal FD channel matrices at the S-D and R-D links, respectively. Furthermore, $\boldsymbol{n}_{0}^{\mathrm{D}, \mathrm{f}}$ and $\overline{\boldsymbol{n}}_{1}^{\mathrm{D} \text {,f }}$ represent the noise vectors having a length of $N$ and represented by $\mathcal{C N}\left(0, \sigma_{N}^{2}\right)$, which are imposed at the BS during the two time-slots, respectively. We amalgamate the above two equations into a $2 N$-length joint observation vector as

$$
\boldsymbol{y}_{k^{\prime}}^{\mathrm{D}, \mathrm{f}}=\sqrt{P_{k^{\prime}}^{\mathrm{S}}} \boldsymbol{H}_{k^{\prime}}^{\mathrm{D}, \mathrm{f}} \boldsymbol{x}_{k^{\prime}}^{\mathrm{f}}+\boldsymbol{n}^{\mathrm{D}, \mathrm{f}},
$$

where the $(2 N \times N)$-element joint equivalent $\mathrm{FD}$ channel matrix is given by

$$
\boldsymbol{H}_{k^{\prime}}^{\mathrm{D}, \mathrm{f}}=\left[\begin{array}{c}
\boldsymbol{H}_{0, k^{\prime}}^{\mathrm{D}, \mathrm{f}} \\
\boldsymbol{H}_{1, k^{\prime}}^{\mathrm{D}, \mathrm{f}}
\end{array}\right]=\left[\begin{array}{c}
\sqrt{\xi_{\mathrm{SD}}} \boldsymbol{H}_{k^{\prime}}^{\mathrm{SD}, \mathrm{f}} \\
\sqrt{G_{\mathrm{RD}} G_{\mathrm{SR}}} \boldsymbol{H}_{k^{\prime}}^{\mathrm{RD}, \mathrm{f}} \boldsymbol{\beta}_{k^{\prime}}^{\mathrm{f}} \boldsymbol{H}_{k^{\prime}}^{\mathrm{SR}, \mathrm{f}}
\end{array}\right] .
$$

Additionally, we formulate the $n$-th element of the diagonal matrices $\boldsymbol{H}_{0, k^{\prime}}^{\mathrm{D}, \mathrm{f}}$ and $\boldsymbol{H}_{1, k^{\prime}}^{\mathrm{D}, \mathrm{f}}$ as

$$
\begin{aligned}
& h_{0, k^{\prime}, n}^{\mathrm{D}, \mathrm{f}}=\sqrt{\xi_{\mathrm{SD}}} h_{S D, k^{\prime} n}^{\mathrm{f}} \\
& h_{1, k^{\prime}, n}^{\mathrm{D}, \mathrm{f}}=\beta_{k^{\prime}, n}^{\mathrm{f}} \sqrt{G_{\mathrm{RD}} G_{\mathrm{SR}}} h_{k^{\prime}, n}^{\mathrm{RD}, \mathrm{f}} h_{k^{\prime}, n}^{\mathrm{SR}, \mathrm{f}} .
\end{aligned}
$$

Similarly, the total received noise of the $k^{\prime}$-th user at the BS includes the noise contribution imposed by the $k^{\prime}$-th relay after the above-mentioned subband remapping operation plus that added at the BS during the two time slots, which is expressed by a $2 N$-length vector as

$$
\boldsymbol{n}^{\mathrm{D}, \mathrm{f}}=\left[\begin{array}{c}
\boldsymbol{n}_{0}^{\mathrm{D}, \mathrm{f}} \\
\overline{\boldsymbol{n}}_{1}^{\mathrm{D}, \mathrm{f}}
\end{array}\right]=\left[\begin{array}{c}
\mathcal{P}_{k^{\prime}}^{T} \mathcal{F}_{U} \tilde{\boldsymbol{n}}_{0}^{\mathrm{D}, \mathrm{t}} \\
\mathcal{P}_{k^{\prime}}^{T} \mathcal{F}_{U}\left(\sqrt{G_{\mathrm{RD}}} \tilde{\boldsymbol{H}}_{k^{\prime}}^{\mathrm{RD}} \overline{\boldsymbol{n}}_{k^{\prime}}^{\mathrm{R}, \mathrm{t}}+\tilde{\boldsymbol{n}}_{1}^{\mathrm{D}, \mathrm{t}}\right)
\end{array}\right] .
$$

\section{B. MMSE assisted Joint Frequency-Domain Equalisation and Combining}

Based on Eq. (12), it can be readily shown that the optimum MMSE solution is given by

$$
\boldsymbol{W}_{k^{\prime}}^{\mathrm{D}}=\left(\boldsymbol{R}_{k^{\prime}}^{\mathrm{yD}}\right)^{-1} \boldsymbol{R}_{k^{\prime}}^{\mathrm{yxD}}
$$

where the auto-correlation matrix of $\boldsymbol{y}_{k^{\prime}}^{\mathrm{D}, \mathrm{f}}$ is given by

$$
\boldsymbol{R}_{k^{\prime}}^{\mathrm{yD}}=P_{k^{\prime}}^{\mathrm{S}} \boldsymbol{H}_{k^{\prime}}^{\mathrm{D}, \mathrm{f}}\left(\boldsymbol{H}_{k^{\prime}}^{\mathrm{D}, \mathrm{f}}\right)^{H}+\boldsymbol{R}_{\mathrm{nD}},
$$

with $\boldsymbol{R}_{\mathrm{nD}}=\mathrm{E}\left[\boldsymbol{n}^{\mathrm{D}, \mathrm{f}}\left(\boldsymbol{n}^{\mathrm{D}, \mathrm{f}}\right)^{H}\right]=\operatorname{diag}\left\{\boldsymbol{R}_{0}^{\mathrm{nD}}, \boldsymbol{R}_{1}^{\mathrm{nD}}\right\}$ denoting the covariance matrix of $n^{\mathrm{D}, \mathrm{f}}$, where $\boldsymbol{R}_{0}^{\mathrm{nD}}=$ $\mathrm{E}\left[\boldsymbol{n}_{0}^{\mathrm{D}, \mathrm{f}}\left(\boldsymbol{n}_{0}^{\mathrm{D}, \mathrm{f}}\right)^{H}\right]=\sigma_{N}^{2} \boldsymbol{I}_{N}$ and $\boldsymbol{R}_{1}^{\mathrm{nD}}=\mathrm{E}\left[\overline{\boldsymbol{n}}_{1}^{\mathrm{D}, \mathrm{f}}\left(\overline{\boldsymbol{n}}_{1}^{\mathrm{D}, \mathrm{f}}\right)^{H}\right]=$ $\sigma_{N}^{2}\left[G_{\mathrm{RD}} \boldsymbol{H}_{k^{\prime}}^{\mathrm{RD}, \mathrm{f}} \boldsymbol{\beta}_{k^{\prime}}^{\mathrm{f}}\left(\boldsymbol{\beta}_{k^{\prime}}^{\mathrm{f}}\right)^{H}\left(\boldsymbol{H}_{k^{\prime}}^{\mathrm{RD}, \mathrm{f}}\right)^{H}+\boldsymbol{I}_{N}\right]$. Thus, the corresponding power of the noise components $\boldsymbol{n}_{0}^{\mathrm{D}, \mathrm{f}}$ and $\overline{\boldsymbol{n}}_{1}^{\mathrm{D}, \mathrm{f}}$ in 
the $n$-th subband during the $\mathrm{TS}_{1}$ and $\mathrm{TS}_{2}$ can be expressed as $\mathcal{N}_{0, n}^{\mathrm{D}}=\sigma_{N}^{2}$, and $\mathcal{N}_{1, n}^{\mathrm{D}}=\sigma_{N}^{2}\left(\beta_{k^{\prime}, n}^{\mathrm{f}}\right)^{2} G_{\mathrm{RD}}\left|h_{k^{\prime}, n}^{\mathrm{RD}, \mathrm{f}}\right|^{2}+\sigma_{N}^{2}$, respectively. In Eq. (16), $\boldsymbol{R}_{k^{\prime}}^{\mathrm{yxD}}$ is the cross-correlation matrix between $\boldsymbol{y}_{k^{\prime}}^{\mathrm{D}, \mathrm{f}}$ and $\boldsymbol{x}_{S, k^{\prime}}^{\mathrm{f}}$, which can be expressed as

$$
\boldsymbol{R}_{k^{\prime}}^{\mathrm{yxD}}=P_{k^{\prime}}^{\mathrm{S}} \boldsymbol{H}_{k^{\prime}}^{\mathrm{D}, \mathrm{f}}
$$

Therefore, when substituting Eq. (17) and Eq. (18) into Eq. (16), the $(2 N \times N)$-element optimum weight matrix of the MMSE aided JFDEC is formulated as

$$
\boldsymbol{W}_{k^{\prime}}^{\mathrm{D}}=P_{k^{\prime}}^{\mathrm{S}}\left[P_{k^{\prime}}^{\mathrm{S}} \boldsymbol{H}_{k^{\prime}}^{\mathrm{D}, \mathrm{f}}\left(\boldsymbol{H}_{k^{\prime}}^{\mathrm{D}, \mathrm{f}}\right)^{H}+\boldsymbol{R}_{\mathrm{nD}}\right]^{-1} \boldsymbol{H}_{k^{\prime}}^{\mathrm{D}, \mathrm{f}},
$$

where the matrix inversion operation can be applied to the $(N \times N)$-element diagonal matrices $\boldsymbol{R}_{0}^{\mathrm{nD}}$ and $\boldsymbol{R}_{1}^{\mathrm{nD}}$, respectively. It is worth noting that $\boldsymbol{R}_{k^{\prime}}^{\mathrm{yD}}$ is an $(2 N \times 2 N)$-element nondiagonal matrix. Hence, the complexity of inverting $\boldsymbol{R}_{k^{\prime}}^{\mathrm{yD}}$ might be high. In order to implement the low complexity singletap FDE and diversity combining jointly, the matrix inversion lemma ${ }^{3}$ of [24] can be invoked, then Eq. (19a) is formulated as

$$
\boldsymbol{W}_{k^{\prime}}^{\mathrm{D}}=P_{k^{\prime}}^{\mathrm{S}} \boldsymbol{R}_{\mathrm{nD}}^{-1} \boldsymbol{H}_{k^{\prime}}^{\mathrm{D}, \mathrm{f}}\left[P_{k^{\prime}}^{\mathrm{S}}\left(\boldsymbol{H}_{k^{\prime}}^{\mathrm{D}, \mathrm{f}}\right)^{H} \boldsymbol{R}_{\mathrm{nD}}^{-1} \boldsymbol{H}_{k^{\prime}}^{\mathrm{D}, \mathrm{f}}+\boldsymbol{I}_{N}\right]^{-1} .
$$

Consequently, by substituting $\boldsymbol{H}_{k^{\prime}}^{\mathrm{D}, \mathrm{f}}$ and $\boldsymbol{R}_{\mathrm{nD}}$ into the above equation, we obtain $W_{k^{\prime}}^{\mathrm{D}}$, which is constituted by two $(N \times N)$-element diagonal matrices, yielding

$$
\begin{aligned}
\boldsymbol{W}_{k^{\prime}}^{\mathrm{D}} & =\left[\begin{array}{c}
\left(\boldsymbol{R}_{0}^{\mathrm{nD}}\right)^{-1} \boldsymbol{H}_{0, k^{\prime}}^{\mathrm{D}, \mathrm{f}} \\
\left(\boldsymbol{R}_{1}^{\mathrm{nD}}\right)^{-1} \boldsymbol{H}_{1, k^{\prime}}^{\mathrm{D}, \mathrm{f}}
\end{array}\right]\left[\left(\boldsymbol{H}_{0, k^{\prime}}^{\mathrm{D}, \mathrm{f}}\right)^{H}\left(\boldsymbol{R}_{0}^{\mathrm{nD}}\right)^{-1} \boldsymbol{H}_{0, k^{\prime}}^{\mathrm{D}, \mathrm{f}}\right. \\
& \left.+\left(\boldsymbol{H}_{1, k^{\prime}}^{\mathrm{D}, \mathrm{f}}\right)^{H}\left(\boldsymbol{R}_{1}^{\mathrm{nD}}\right)^{-1} \boldsymbol{H}_{1, k^{\prime}}^{\mathrm{D}, \mathrm{f}}+1 / P_{k^{\prime}}^{\mathrm{R}} \boldsymbol{I}_{N}\right]^{-1} .
\end{aligned}
$$

Hence the $n$-th and $(n+N)$-th element of $\boldsymbol{W}_{k^{\prime}}^{\mathrm{D}}$ can be expressed by

$$
w_{k^{\prime}, n}^{\mathrm{D}}=h_{0, k^{\prime}, n}^{\mathrm{D}, \mathrm{f}} e_{k^{\prime} n} / \sigma_{N}^{2}, \quad w_{k^{\prime},(n+N)}^{\mathrm{D}}=h_{1, k^{\prime}, n}^{\mathrm{D}, \mathrm{f}} e_{k^{\prime} n} / \mathcal{N}_{1, n}^{\mathrm{D}},
$$

where $e_{k^{\prime} n}$ is given by

$$
e_{k^{\prime} n}=\left(\left|h_{0, k^{\prime}, n}^{\mathrm{D}, \mathrm{f}}\right|^{2} / \sigma_{N}^{2}+\left|h_{1, k^{\prime}, n}^{\mathrm{D}, \mathrm{f}}\right|^{2} / \mathcal{N}_{1, n}^{\mathrm{D}}+1 / P_{k^{\prime}}^{\mathrm{R}}\right)^{-1} .
$$

Actually, $e_{k^{\prime} n}$ is the FD MMSE value in the $n$-th subband of the $k^{\prime}$-th user's signal, which will be discussed in more detail in Subsection III-C. It will be shown that the weight matrix $W_{k^{\prime}}^{\mathrm{D}}$ is capable of carrying out single-tap FDE and diversity combining of the direct and relay branches jointly. Specifically, the coefficients $h_{0, k^{\prime}, n}^{\mathrm{D}, \mathrm{f}} e_{k^{\prime} n}$ and $h_{1, k^{\prime}, n}^{\mathrm{D}, \mathrm{f}} e_{k^{\prime} n}$ in Eq. (20) are used for single-tap FDE in conjunction with diversity combining, while obeying the MMSE criterion, where noise whitening is carried out at both branches by normalising it according to the noise power $\sigma_{N}^{2}$ and $\mathcal{N}_{1, n}^{\mathrm{D}}$, respectively.

\section{Relationships of MMSE, SNR and SINR}

Correspondingly, upon applying the weight matrix $\left(\boldsymbol{W}_{k^{\prime}}^{\mathrm{D}}\right)^{H}$ to $\boldsymbol{y}_{k^{\prime}}^{\mathrm{D}, \mathrm{f}}$, the signals arriving from both the direct and relaying branches are combined into a $N$-length observation vector

\footnotetext{
${ }^{3}$ Assuming that the matrices $\boldsymbol{A}$ and $\boldsymbol{B}$ have $(N \times N)$ and $(N \times M)$ elements, respectively, and $\boldsymbol{I}_{M}$ is an $(M \times M)$-element identity matrix, we have $\left(\boldsymbol{A}+\boldsymbol{B} \boldsymbol{B}^{H}\right)^{-1} \boldsymbol{B}=\boldsymbol{A}^{-1} \boldsymbol{B}\left(\boldsymbol{B}^{H} \boldsymbol{A}^{-1} \boldsymbol{B}+\boldsymbol{I}_{M}\right)^{-1}$.
}

in the FD, which are then transformed into the TD decision variable vector for $\boldsymbol{x}_{k^{\prime}}^{\mathrm{t}}$ by the $N$-point IDFT, yielding

$$
\hat{\boldsymbol{y}}_{k^{\prime}}^{\mathrm{D}, \mathrm{t}}=\boldsymbol{F}_{N}^{H}\left(\boldsymbol{W}_{k^{\prime}}^{\mathrm{D}}\right)^{H} \boldsymbol{y}_{k^{\prime}}^{\mathrm{D}, \mathrm{f}}=\boldsymbol{A}_{k^{\prime}}^{\mathrm{t}} \boldsymbol{x}_{k^{\prime}}^{\mathrm{t}}+\hat{\boldsymbol{n}}_{D}^{\mathrm{t}},
$$

where $\boldsymbol{A}_{k^{\prime}}^{\mathrm{t}}$ is the $(N \times N)$-element TD circulant influence matrix of desired signals and $\hat{\boldsymbol{n}}_{D}^{\mathrm{t}}$ is the $N$-length TD noise vector after equalisation. The gain factor between $x_{k^{\prime}}^{\mathrm{t}}$ and its estimate of $\hat{\boldsymbol{y}}_{k^{\prime}}^{\mathrm{D}, \mathrm{t}}$ is given by the diagonal elements of the matrix $A_{k^{\prime}}^{\mathrm{t}}$ [43].

Furthermore, the TD estimation error vector $\boldsymbol{e}_{k^{\prime}}^{\mathrm{t}}$ between the transmitted signal $\boldsymbol{x}_{k^{\prime}}^{\mathrm{t}}$ and the estimated signal $\hat{\boldsymbol{y}}_{k^{\prime}}^{\mathrm{D}, \mathrm{t}}$ can be expressed as

$$
\boldsymbol{e}_{k^{\prime}}^{\mathrm{t}}=\boldsymbol{x}_{k^{\prime}}^{\mathrm{t}}-\hat{\boldsymbol{y}}_{k^{\prime}}^{\mathrm{D}, \mathrm{t}}=\boldsymbol{x}_{k^{\prime}}^{\mathrm{t}}-\boldsymbol{A}_{k^{\prime}}^{\mathrm{t}} \boldsymbol{x}_{k^{\prime}}^{\mathrm{t}}-\hat{\boldsymbol{n}}_{D}^{\mathrm{t}},
$$

where the covariance matrix of $\boldsymbol{e}_{k^{\prime}}^{\mathrm{t}}$ is given by

$$
\begin{aligned}
\boldsymbol{R}_{e_{k^{\prime}}^{\mathrm{t}}} & =\mathrm{E}\left[\boldsymbol{e}_{k^{\prime}}^{\mathrm{t}}\left(\boldsymbol{e}_{k^{\prime}}^{\mathrm{t}}\right)^{H}\right] \\
& =P\left[\boldsymbol{I}_{N}-2 \Re\left\{A_{k^{\prime}}^{\mathrm{t}}\right\}+\boldsymbol{A}_{k^{\prime}}^{\mathrm{t}}\left(\boldsymbol{A}_{k^{\prime}}^{\mathrm{t}}\right)^{H}\right]+\sigma_{N}^{2} \boldsymbol{I}_{N},
\end{aligned}
$$

with $\Re\left\{\boldsymbol{A}_{k^{\prime}}^{\mathrm{t}}\right\}$ denoting the real part of $\boldsymbol{A}_{k^{\prime}}^{\mathrm{t}}$. Therefore, $\boldsymbol{R}_{e^{\mathrm{t}}}$ is also a $(N \times N)$-element circulant matrix, having identical diagonal elements, which implies that, due to the averaging effects of the $N$-point IDFT operation, all the $k$-th users' resultant TD symbols within the vector have the same MMSE value $e_{k^{\prime}}$. This MMSE value can be calculated as the average of the FD MMSE values over $N$ subbands, yielding the MMSE of the joint solution as

$$
e_{k^{\prime}}=\frac{1}{N} \sum_{n=0}^{N-1} e_{k^{\prime}, n}=\frac{1}{N} \operatorname{Tr}\left[\boldsymbol{R}_{e_{k^{\prime}}^{\mathrm{f}}}\right] .
$$

Hence the Eq. (21) can be also derived by

$$
\begin{aligned}
e_{k^{\prime}, n} & =\left[\boldsymbol{R}_{e_{k^{\prime}}^{\mathrm{f}}}\right]_{n}=\left[P_{k^{\prime}}^{\mathrm{S}} \boldsymbol{I}_{N}-\left(\boldsymbol{R}_{k^{\prime}}^{\mathrm{yxD}}\right)^{H} \boldsymbol{W}_{k^{\prime}}^{\mathrm{D}}\right]_{n} \\
& =P_{k^{\prime}}^{\mathrm{S}} /\left(\gamma_{k^{\prime}, n}^{\mathrm{D} 0}+\gamma_{k^{\prime}, n}^{\mathrm{D} 1}+1\right),
\end{aligned}
$$

where the instantaneous received SNRs of the direct and relaying branches in the $n$-th subband are expressed as

$$
\begin{aligned}
\gamma_{k^{\prime}, n}^{\mathrm{D} 0} & =\gamma_{k^{\prime}, n}^{\mathrm{SD}}=P_{k^{\prime}}^{\mathrm{S}} \xi_{\mathrm{SD}}\left|h_{S D, k^{\prime} n}^{\mathrm{f}}\right|^{2} / \sigma_{N}^{2}, \\
\gamma_{k^{\prime}, n}^{\mathrm{D} 1} & =\left(1 / \gamma_{k^{\prime}, n}^{\mathrm{SR}}+1 / \gamma_{k^{\prime}, n}^{\mathrm{RD}}\right)^{-1}, \\
\gamma_{k^{\prime}, n}^{\mathrm{SR}} & =P_{k^{\prime}}^{\mathrm{S}} G_{\mathrm{SR}}\left|h_{k^{\prime}, n}^{\mathrm{SR}, \mathrm{f}}\right|^{2} / \sigma_{N}^{2}, \\
\gamma_{k^{\prime}, n}^{\mathrm{RD}} & =\frac{P_{k^{\prime}}^{\mathrm{R}} G_{\mathrm{RD}} G_{\mathrm{SR}}\left|h_{k^{\prime}, n}^{\mathrm{RD}, \mathrm{f}}\right|^{2}\left|h_{k^{\prime}, n}^{\mathrm{SR}, \mathrm{f}}\right|^{2}}{\sigma_{N}^{2}\left(G_{\mathrm{SR}}\left|h_{k^{\prime}, n}^{\mathrm{SR}, \mathrm{f}}\right|^{2}+\sigma_{N}^{2} / P_{k^{\prime}}^{\mathrm{S}}\right)} .
\end{aligned}
$$

We note that $e_{k^{\prime}, n}$ has already appeared in Eq. (21) and it is also equivalent to the general result for the optimum MMSE solution of the non-cooperative scenario [24], [44].

Consequently, according to the relationship between the SINR and MMSE as a general feature of the MMSE criterion [24], we obtain the $k^{\prime}$-th user's instantaneous overall received SINR at the output of our proposed scheme in the form of

$$
\begin{aligned}
\gamma_{k^{\prime}} & =P_{k^{\prime}}^{\mathrm{S}} e_{k^{\prime}}^{-1}-1 \\
& =\left\{\frac{1}{N} \sum_{n=0}^{N-1}\left[\begin{array}{c}
1+\gamma_{k^{\prime}, n}^{\mathrm{SD}}+ \\
\left(1 / \gamma_{k^{\prime}, n}^{\mathrm{SR}}+1 / \gamma_{k^{\prime}, n}^{\mathrm{RD}}\right)^{-1}
\end{array}\right]^{-1}\right\}^{-1}-1,
\end{aligned}
$$

which is derived in the Appendix in the form of Eq. (45). 


\section{Energy Consumption Metrics}

In order to evaluate the multi-user system's performance in terms of the achievable power reduction, we introduce two energy consumption metrics, namely the Energy Consumption Rate (ECR) expressed in the unit of Joule per bit and the Energy Consumption Gain (ECG), which is defined as the ratio of the ECR of reference system over that of the system advocated [41]. Therefore, the ECR of single-hop direct transmission operating without cooperation may be defined as

$$
E C R_{\mathrm{dir}}=\sum_{k=0}^{K-1} \frac{P_{k} T}{R_{k}^{\mathrm{dir}} T}=\sum_{k=0}^{K-1} \frac{P_{k}}{R_{k}^{\mathrm{dir}}}=\frac{K P}{R_{\Sigma}^{\mathrm{dir}}},
$$

where $T$ denotes the time-slot duration, i.e. time duration per hop, while $R_{k}^{(\cdot)} R_{\Sigma}^{(\cdot)}$ denote the achievable ergodic single-user rate and multi-user sum-rates of the $(\cdot)$-type transmission, i.e. of direct or of AF-cooperative transmissions.

Similarly, the ECR of two-hop AF-cooperative transmissions may be expressed as

$$
E C R_{\mathrm{AF}}=\sum_{k=0}^{K-1} \frac{P_{k}^{\mathrm{S}} T+P_{k}^{\mathrm{R}} T}{R_{k}^{\mathrm{AF}} 2 T}=\sum_{k=0}^{K-1} \frac{P_{k}^{\mathrm{S}}+P_{k}^{\mathrm{R}}}{2 R_{k}^{\mathrm{AF}}} .
$$

Since we assume $\sum_{k=0}^{K-1}\left(P_{k}^{\mathrm{S}}+P_{k}^{\mathrm{R}}\right)=K P$, by using the direct transmission as a reference, the ECG of cooperative transmissions is given by

$$
E C G=\frac{E C R_{\mathrm{dir}}}{E C R_{\mathrm{AF}}}=\frac{2 R_{\Sigma}^{\mathrm{AF}}}{R_{\Sigma}^{\mathrm{dir}}} .
$$

We assume that the system bandwidth was normalised to unity in our baseband processing, hence $R_{\Sigma}^{(\cdot)}$ expressed in terms of bits $/ \mathrm{sec} / \mathrm{Hz}$ is given by

$$
R_{\Sigma}^{(\cdot)}=\mathrm{E}\left[\sum_{k=0}^{K-1} \frac{1}{N_{\mathrm{TS}}} \log _{2}\left(1+\gamma_{k}^{(\cdot)}\right)\right],
$$

where the factor $1 / N_{\mathrm{TS}}$ indicates the effect of the $N_{\mathrm{TS}}$ timeslots required for direct or cooperative transmission.

Therefore, the corresponding instantaneous SINR of the $k$ th user may be expressed as

$$
\gamma_{k}^{(\cdot)}=\left[\frac{1}{N} \sum_{n=0}^{N-1}\left(1+\gamma_{k, n}^{(\cdot)}\right)^{-1}\right]^{-1}-1 .
$$

Specifically, for direct transmission, we have an equivalent instantaneous SNR in the $n$-th subband of the $k$-th user given by

$$
\gamma_{k, n}^{\operatorname{dir}}=P_{k} \xi_{\text {dir }}\left|h_{k, n}^{\text {dir,f }}\right|^{2} / \sigma_{N}^{2}
$$

By contrast, for AF-cooperation, the the $k$-th user's received equivalent instantaneous SNR in the $n$-th subband can be expressed as according to Eq. (28),

$$
\gamma_{k, n}^{\mathrm{AF}}=\gamma_{k, n}^{\mathrm{SD}}+\left[\left(\gamma_{k, n}^{\mathrm{SR}}\right)^{-1}+\left(\gamma_{k, n}^{\mathrm{RD}}\right)^{-1}\right]^{-1}
$$

\section{OpPORTUNISTIC COOPERATIVE RELAYING}

The opportunistic relaying allows a single relay to be selected from a cluster of $J(J>0)$ inactive MTs, which are the RCs, depending on which MT provides the best endto-end link between the source and destination [12]. In this section, the source/relay power sharing and relay selection are investigated for the sake of power-efficient opportunistic cooperation. Three relay selection schemes, namely the SURS, MU-RS and MA-RS regimes, are shown in Fig. 3. The BS is assumed to be capable of acquiring the Channel State Information at Receiver (CSIR) and the SNR of all the cooperative links based on pilot-assisted channel estimation, which were formulated in Eq. (27a), (27c) and (27d). The power sharing and relay selection are carried out with the objective of maximising the average received instantaneous SINR of each user at the BS for both the direct and relaying branches. Additionally, we assume that the transmissions of the S-D, S-R and R-D links are orthogonal and hence they do not impose an increased MUI.
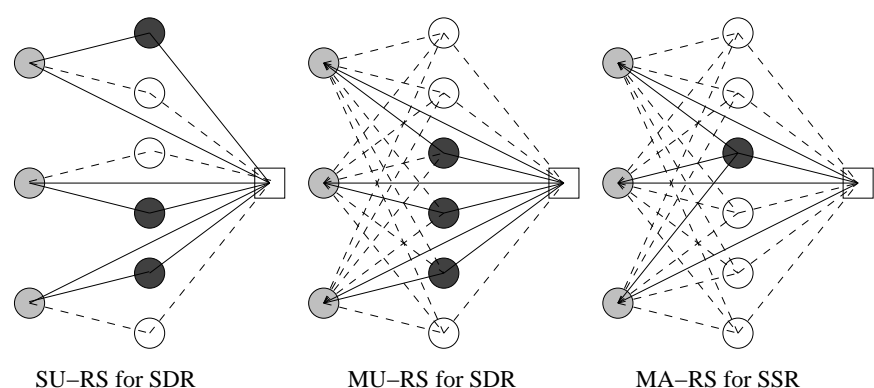

Source $\bigcirc$ Relay Candidate

Target Relay Base Station

Fig. 3. Opportunistic Cooperative Relaying aided SC-FDMA Uplink

\section{A. Source/Relay Power Allocation}

The proposed systems invoke the linear and adaptive source/relay power sharing modes. The family of linear power sharing includes Equal Power Allocation (EPA) and Default Power Allocation (DPA) for the SDR and SSR systems, respectively. Specifically, the EPA mode adjusts the transmitted powers of the $k$-th source and $j$-th RC to be equal, while the DPA mode shares the transmitted power between the source MT and relay according to the number of active source users within system. Hence we have

$$
\begin{array}{ll}
\text { EPA for SDR : } & \alpha_{j, k}^{\mathrm{S}}=\alpha_{j, k}^{\mathrm{R}}=0.5 \\
\text { DPA for SSR : } & \alpha_{j, k}^{\mathrm{S}}=1-1 / K, \\
& \alpha_{j, k}^{\mathrm{R}}=1 / K .
\end{array}
$$

Alternatively, the adaptive OPA mode may be invoked for both SDR and SSR systems, which maximises the average SINR expression of Eq. (28) for the $k$-th user with the aid of the 
$j$-th RC by associating the CSIR, yielding

$$
\begin{aligned}
\max _{\alpha_{j, k}^{\mathrm{S}}, \alpha_{j, k}^{\mathrm{R}}} & \gamma_{j, k}^{\mathrm{AF}}\left(\alpha_{j, k}^{\mathrm{S}}, \alpha_{j, k}^{\mathrm{R}}\right) \\
\text { subject to } & \alpha_{j, k}^{\mathrm{S}}+\alpha_{j, k}^{\mathrm{R}}=1, \\
& \alpha_{j, k}^{\mathrm{S}}>0, \quad \alpha_{j, k}^{\mathrm{R}}>0, \\
& \text { for SSR }: \sum_{k=0}^{K-1} \alpha_{j, k}^{\mathrm{R}}=1 .
\end{aligned}
$$

\section{B. Single-User Relay Selection}

Initially, we examine the relay selection schemes designed for the SDR topology. If the total number of idle MTs accessing the single-cell BS is high, more RCs may be considered for activation as the target relay. Since each source MT is capable of seeking a target relay from a cluster of $J$ RCs, which are independent from the other source MT's RC, we propose the SU-RS scheme, where a total of $(K \times J)$ inactive MTs are required in order to support $K$ source MTs. The BS calculates the overall SINR of the $k$-th user, while tentatively relying on any of the $J \mathrm{RCs}$ and then selects the one having maximum SINR, which is associated with the index of $j_{k}^{\text {opt }}$, as formulated in

$$
j_{k}^{\mathrm{opt}}=\arg \max _{j \in[0, J-1]}\left\{\gamma_{j, k}^{\mathrm{AF}}\right\}, \quad(J>0) .
$$

\section{Multi-User Relay Selection}

However, when the total number of inactive MTs roaming within a cell is low, the number of available RCs may become insufficient, since $J \times K$ RCs would be required for $K$ sources in SU-RS. In order to circumvent this limitation of the SDR regime, we propose the MU-RS scheme, which allows the multiple source users to access a shared RC, where each source's data is forwarded by selecting a single desired relay for cooperative transmission. Specifically, a cluster of $K$ source MTs is collectively assocated with a cluster of $J(J \geq K)$ $\mathrm{RCs}$, but the system only requires a total of $K$ target relays. Furthermore, the system invokes the optimal partner ordering method by extending the relay ordering regime of [16] in order to take into account both multiple sources and multiple relays, in order to optimise the cooperative partner pairing. Our regime calculates the overall SINR of all the $K$ source MTs' signal by tentatively assuming cooperation with all the $J$ RCs, and chooses the specific source relay pairs having the highest $K$ received SINR values at the BS, which are provided by the RCs corresponding to the particular source MTs. Specifically, at the $i$-th iteration, the desired relay's index $j_{k, i}^{\text {opt }}$ selected for assisting the $k$-th source MT is assumed to be the pairing index $i$, which are compiled in descending order, yielding

$$
j_{k, i}^{\text {opt }}=\arg \max _{j \in[0, J-1], k \in[0, K-1]}\left\{\gamma_{j, k}^{\mathrm{AF}}\right\}, \quad(J \geq K) .
$$

Thus, upon removing the $k$-th source MT and the $j_{k, i}^{\text {opt }}$-th relay from the selected pools, the target relay of the $(k+1)$-st user may be allocated during the next iteration using Eq. (39).

\section{Multiple-Access Relay Selection}

By contrast, in the SSR system, the relay selection should take into account the overall performance of all the links from all source MTs aiming to share a relay, because multiple source MTs have to gain access to a common target relay by evaluating the benefits of an entire cluster of RCs, where the minimum number of RCs is independent of the number of sources, i.e. we have $J>0$. Hence, the proposed MA-RS scheme looks for a single relay indicated by $j^{\text {opt }}$, which offers the maximum sum of SINRs results for the $K$ source MTs, which is formulated as:

$$
j^{\mathrm{opt}}=\arg \max _{j \in[0, J-1]}\left\{\gamma_{j, \Sigma}=\sum_{k=0}^{K-1} \gamma_{j, k}^{\mathrm{AF}}\right\}, \quad(J>0) .
$$

\section{E. Computational Complexity}

The computational complexity of the different power allocation and relay selection procedures should be quantified in order to find the most suitable algorithm. The EPA benefits from the lowest complexity, regardless of the number of users and relays involved, while the DPA requires both the sources and the relays to have the knowledge of $K$, but beneficially it dispenses with any iterations. The OPA exhibits the highest complexity, which depends on both the specific optimisation algorithm employed as well as on the stepsize of the power control strategy adopted. From a MU point of view, the SURS, MU-RS and MA-RS all require $(K \times J)$ iterations among RCs for all $K$ users. However, from a SU point of view, the SU-RS only needs $J$ iterations, which is independent of the number of sources, while the MU-RS still requires $(K \times J)$ iterations to calculate the index of the desired relay for each source MT. Additionally, the RRS does not require any iterations among RCs. We assume that in practical cellular systems, the number of cooperative users and the number of RCs within each clusters may be not high, say less than 10 in total, but lower-complexity search algorithms may be considered for this selection stage in order to reduce the signal processing power and time delay.

\section{Simulation Results and Discussions}

In our simulations, we investigate the uncoded Binary Phase Shift Keying (BPSK) modulated link-level performance of the proposed schemes subject to shadowing under imperfect power control with propagation path-loss scenario, experiencing the frequency-selective Rayleigh fading. Our parameters are summarised in Table I.

TABLE I

SIMULATION PARAMETERS

\begin{tabular}{|l|l|}
\hline Subband mapping scheme & Interleaved \\
\hline Number of source users & $K=4,8$ \\
\hline Total number of relay candiates & 16 or varying \\
\hline Number of subbands per user & $N=8$ \\
\hline Bandwidth expansion factor & $M=8$ \\
\hline Total number of subbands & $U=64$ \\
\hline Number of paths & $L=8$ \\
\hline Path-loss exponent & $\eta=0,4$ \\
\hline Shadowing variance & $\sigma_{\xi}^{2}=0,2,4,8(\mathrm{~dB})$ \\
\hline PCE variance & $\sigma_{\epsilon}^{2}=0,2(\mathrm{~dB})$ \\
\hline
\end{tabular}


Initially, we consider a simple idealised system experiencing small-scale fading only, while the effects of shadowing, path-loss, relay selection and power control are all ignored. Fig. 4 illustrates the Bit Error Ratio (BER) versus $E_{b} / N_{0}$ performance of the AF relaying system relying on different receiver solutions for full-load uplink transmissions over a dispersive channel having $L=8$ paths, while supporting $K=8$ users. Compared to the FDE-EGC method, the proposed JFDEC scheme carries out the FDE and diversity combining jointly with the aid of the optimised weights of Eq. (20) over each subband for both the direct and relaying branches. Quantitatively, our proposed scheme is capable of achieving an approximately $4 \mathrm{~dB}$ power reduction at a BER of $10^{-4}$, which is an explicit benefit of the cooperative diversity gain, while the FDE-EGC scheme only saves $2 \mathrm{~dB}$ power.

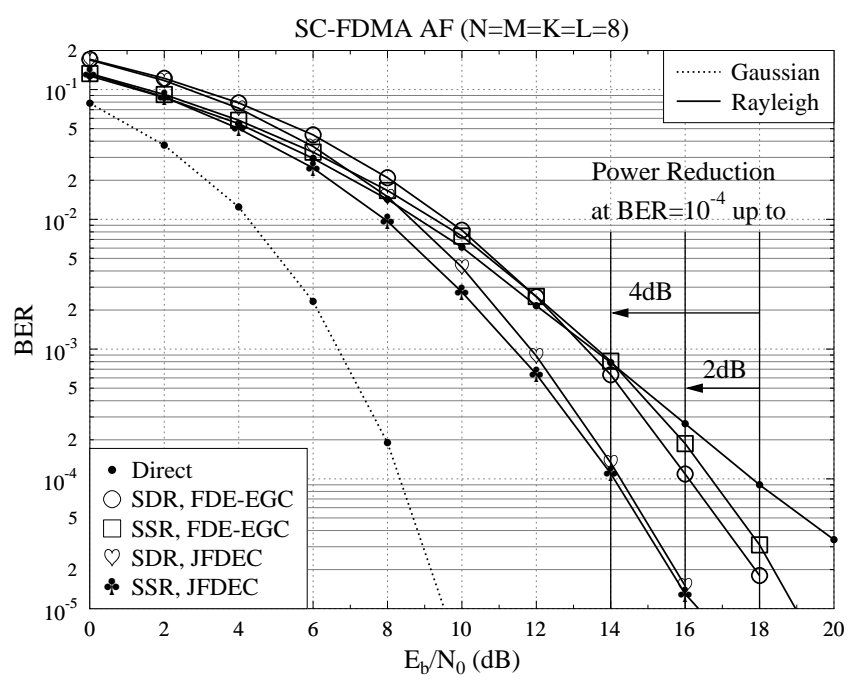

Fig. 4. BER performance comparison and power reduction by ignoring the effects of path-loss and shadowing

Furthermore, in order to characterise the effects of varying the ratio of the source/relay power and the locations of the relays in the presence of path-loss but without shadowing, Fig. 5 depicts the BER versus $\left(\alpha_{k}^{\mathrm{S}}, \delta_{\mathrm{RD}}\right)$ performance of the SDR toplogy for $\eta=4$ at $E_{b} / N_{0}=8 \mathrm{~dB}$, where perfect power control is assumed for both the source and relay. Since the path-loss of the S-R and R-D links is lower than that of the S-D link, high relaying gains are attainable at the BS via the relaying branch, which is confirmed by the curve denoted by the $\vee$ marker. Since we assume that the total transmitted power of the system is constant, the source MT can transmit its own signal at a higher $\alpha_{k}^{\mathrm{S}}$, as indicated by the curve marked by $\wedge$ for $\alpha_{k}^{\mathrm{S}}=0.75$, which is then attenuated by the longdistance S-D and S-R link during $\mathrm{TS}_{1}$. By contrast, the relay transmits the signal forwarded to the destination at a lower $\alpha_{k}^{\mathrm{R}}$ in $\mathrm{TS}_{2}$, when the relay is roaming within the desired relaying area near to the BS, as indicated by the curve marked by legend $\vee$ for $\delta_{\mathrm{RD}}=0.4$, which is expected to have a high relaying gain. Therefore, observing the relay located at this optimum position, the BER performance of the EPA mode recorded for $\alpha_{k}^{\mathrm{S}}=0.5$ only slightly decreases comparing to the OPA mode for $\alpha_{k}^{\mathrm{S}}=0.75$, but requires a lower complexity. Additionally, due to the power constraint of the SSR uplink, the default transmit power of the source MT is a function of $K$. Hence, the optimum value of $\alpha_{k}^{\mathrm{S}}$ may be the $\alpha_{k}^{\mathrm{S}}$ of DPA for the SSR topology, which is larger than the optimum value of $\alpha_{k}^{\mathrm{S}}$ designed for the SDR topology. In summary, both the EPA and DPA are more practical schemes when subjected to imperfect power control, which is an explicit benefit of their low complexity, when invoked in the context of the SDR and SSR topologies, respectively. By contrast, the OPA improves the achievable performance at an increased complexity.

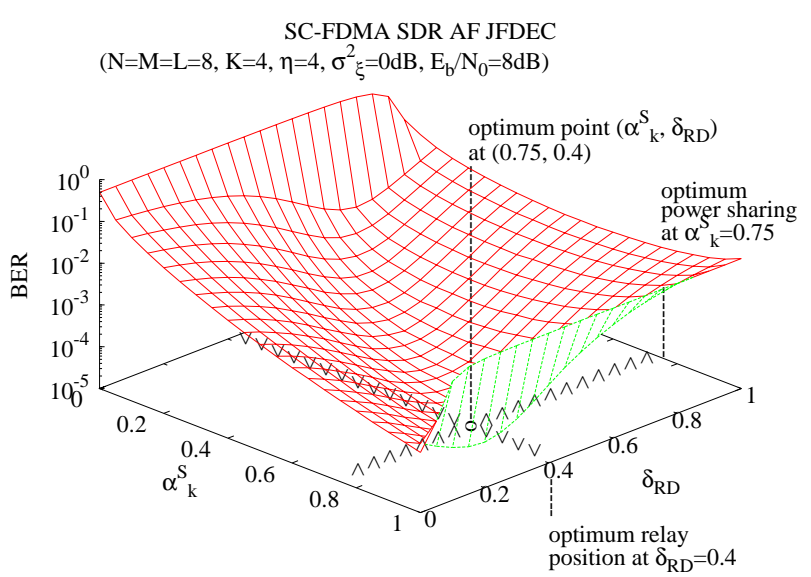

Fig. 5. Effects upon varying shared power and relaying distance

For the sake of fair comparsions, we assume that we have $K=4$ source MTs, assisted by $J=4$ and $J=16$ RCs for SU-RS and MU-RS, respectively. Upon varying the effects of shadowing under imperfect power control at all links for transmission over Rayleigh fading channels in the presence of path-loss for $\eta=4$, Fig. 6 and 7 characterises the overall average BER versus $E_{b} / N_{0}$ performance of different relay selection aided AF systems invoking EPA and OPA, respectively. Observe in Fig. 7 that the achievable gain of the OPA recorded for $\alpha_{k}^{\mathrm{S}}=0.75$ is limited to about $1 \mathrm{~dB}$ at a BER of $10^{-4}$ compared to the EPA curves recorded for $\alpha_{k}^{\mathrm{S}}=0.5$ in Fig. 6, which indicates the robustness of the proposed cooperative regime. We know additionally that according to Fig. 5, the activation of the relay in the vicinity of the optimal location is capable of achieving a similar performance to OPA. Compared to the RRS characterised in both Fig. 6 and 7, the fixed relay associated with the optimal relay position of $\delta_{\mathrm{RD}}=0.4$ offers a $3 \mathrm{~dB}$ relaying gain at a BER of $10^{-4}$, while both SU-RS and MU-RS provide a substantial multiuser diversity gain, which is about $4 \mathrm{~dB}$ at a BER of $10^{-4}$. Furthermore, the proposed MU-RS has the edge over the SU$\mathrm{RS}$, providing an additional multi-user diversity gain in excess of $2 \mathrm{~dB}$, which is a benefit of the relay selection procedure that avoids the effects of deep shadow fading. As a result, the transmitted power can be reduced by about $\left(E_{b} / N_{0}\right)_{\Delta}=4 \mathrm{~dB}$ at a BER of $10^{-4}$, when we have $\sigma_{\xi}^{2}=4 \mathrm{~dB}, \sigma_{\epsilon}^{2}=0 \mathrm{~dB}$.

By contrast, the relay selection effects on the attainable BER performance of the SSR system upon varying the shadow fading variance under imperfect power control are charac- 


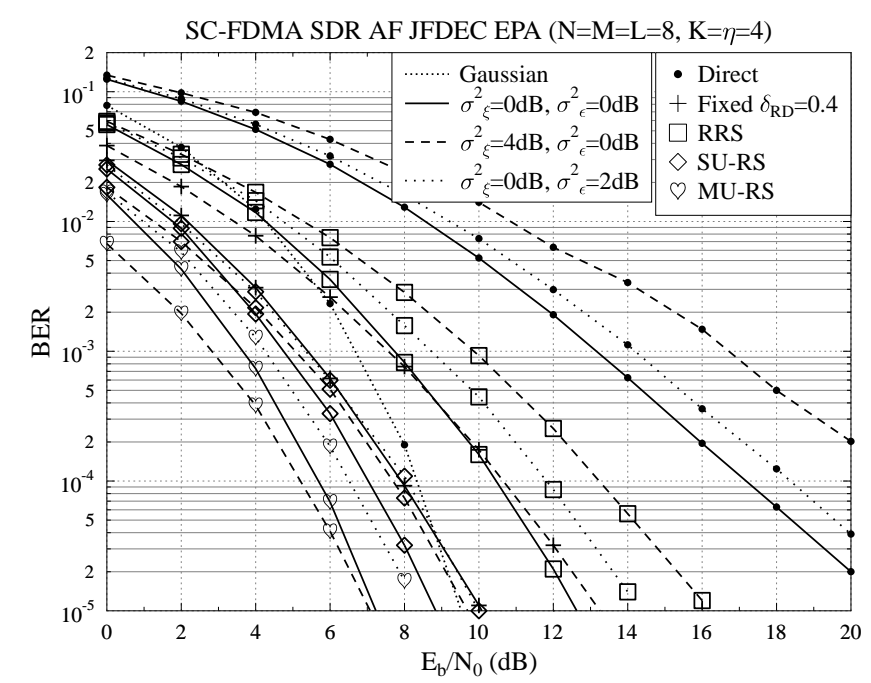

Fig. 6. SDR: BER performance of relay selection schemes invoking EPA

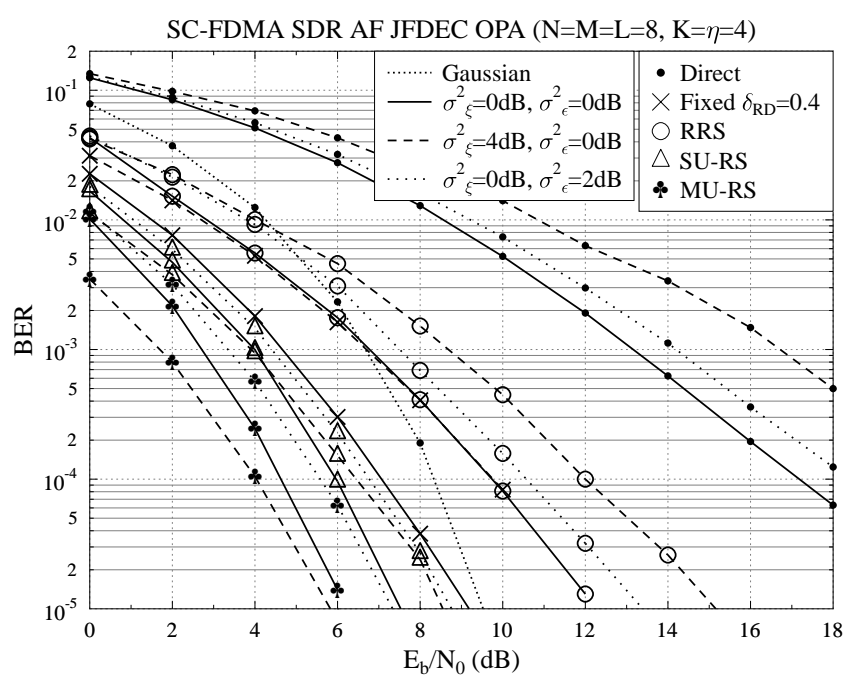

Fig. 7. SDR: BER performance of relay selection schemes invoking OPA

terised in Fig. 8. The fixed relay is capable of obtaining a $3 \mathrm{~dB}$ relaying gain, which is similar to that of the SDR system. Although only a single R-D link exists in system, this target relay can be selected by considering the position trade-offs among multiple source MTs and multiple RCs, in an effort to reduce the path-loss. Meanwhile, a useful diversity gain is still attainable by relay selection for transmission over Rayleigh fading channels. However, the MA-RS considers the single-relay based support of all $K$ source users, whose signal occupies all the $(K \times N)$ subbands, while considering all the $(K \times J)$ possible $\mathrm{S}-\mathrm{R}$ links. Thus, the selection diversity gain achieved in the presence of deep shadow fading does not improve the link-level reliability quantified in terms of the BER performance. Therefore, the DPA aided MA-RS allows the system to achieve a BER of $10^{-4}$ at $E_{b} / N_{0}=6 \mathrm{~dB}$ when we have $\sigma_{\xi}^{2}=\sigma_{\epsilon}^{2}=0 \mathrm{~dB}$. Additionally, since the power control determines the transmit power of all source MTs and relays, the corresponding performance differences between $\sigma_{\epsilon}^{2}=0$ and $\sigma_{\epsilon}^{2}=2$ are quantified in Fig. 6-8 for the EPA, OPA and DPA schemes in the presence of both perfect and imperfect power control.

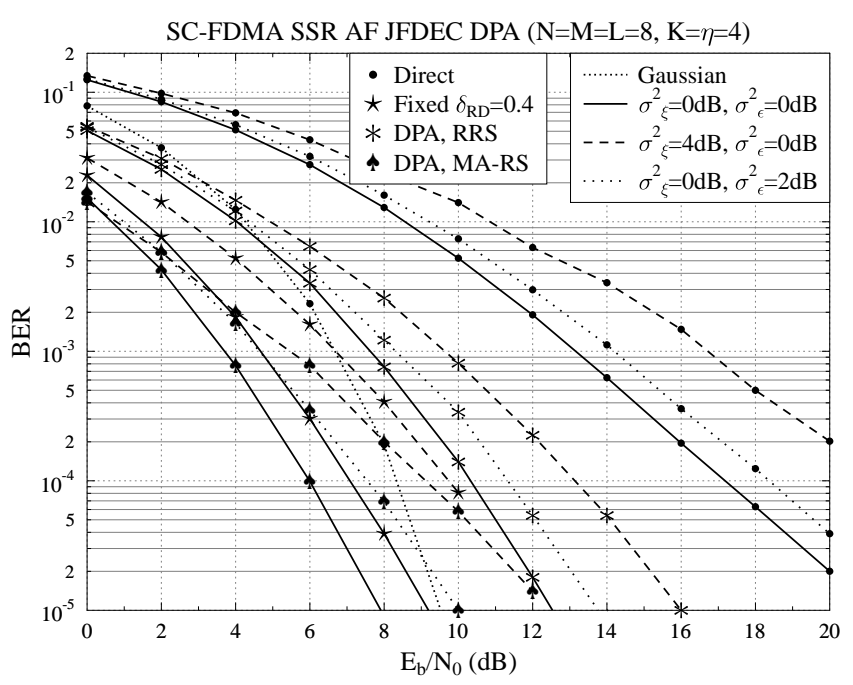

Fig. 8. SSR: BER performance of relay selection schemes invoking DPA

In addition to the above BER performance analysis, we quantify the sum-rate of all user's data transmissions in order to represent the attainable multi-user system throughput in terms of Shannon's Continuous-input Continuous-output Memoryless Channel (CCMC) capacity [45]. From a resource managemet point of view, Fig. 9 depicts the attainable system throughput as a function of the total number of RCs, when the number of source MTs was fixed to $K=4$ and we had $E_{b} / N_{0}=4 \mathrm{~dB}$. Observe in Fig. 9 that the sum-rate improves upon increasing the number of RCs. Specifically, the MU-RS scheme is capable of substantially improving the throughput compared to the SU-RS approach, particularly when communicating over channels subject to shadow fading. Moreover, the sum-rate of the MA-RS is not unduly affected by varying the number of RCs, when compared to both the SU-RS and MU-RS schemes. Additionally, we note that the MA-RS is capable of supporting opportunistic relaying, even when the number of RCs is $J<K$.

For the sake of quantifying the attainable energy-efficiency, when invoking different power sharing and relay selection schemes, Fig. 10 illustrates the ECG versus the $E_{b} / N_{0}$ performance of both the SDR and SSR systems for a shadowing variance of $8 \mathrm{~dB}$ under perfect power control for the SURS in conjunction with $J=4$ and for the MU-RS using $J=16$. Clearly, when aiming for a fixed target $E_{b} / N_{0}$ of say $-10 \mathrm{~dB}$ in the presence of shadowing compared to a non-cooperative direct transmission scenario, the multi-user diversity gain accrued from avoiding small-scale fading allows the MU-RS scheme to achieve a significant ECG of 4 for EPA and 4.5 for OPA, while the SU-RS attains gains of 2.6 and 3.1 for EPA and OPA, respectively. The MA-RS obtains a similar ECG as the OPA aided SU-RS, but imposes a 


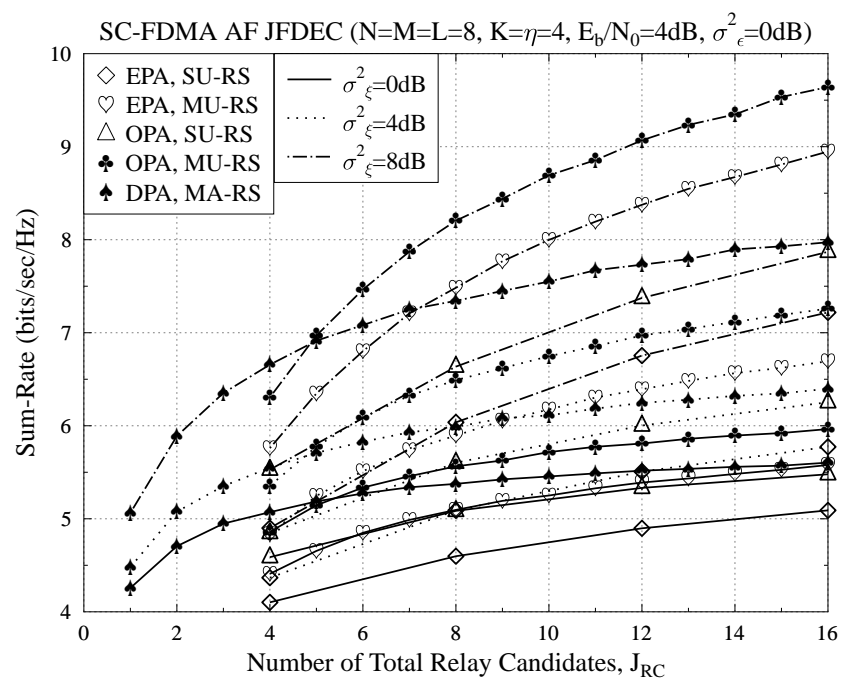

Fig. 9. Effect of varying the number of relay candidates

significantly lower complexity. Finally, the RRS has the lowest gain of about 1.5 , regardless of the specific power allocation employed.

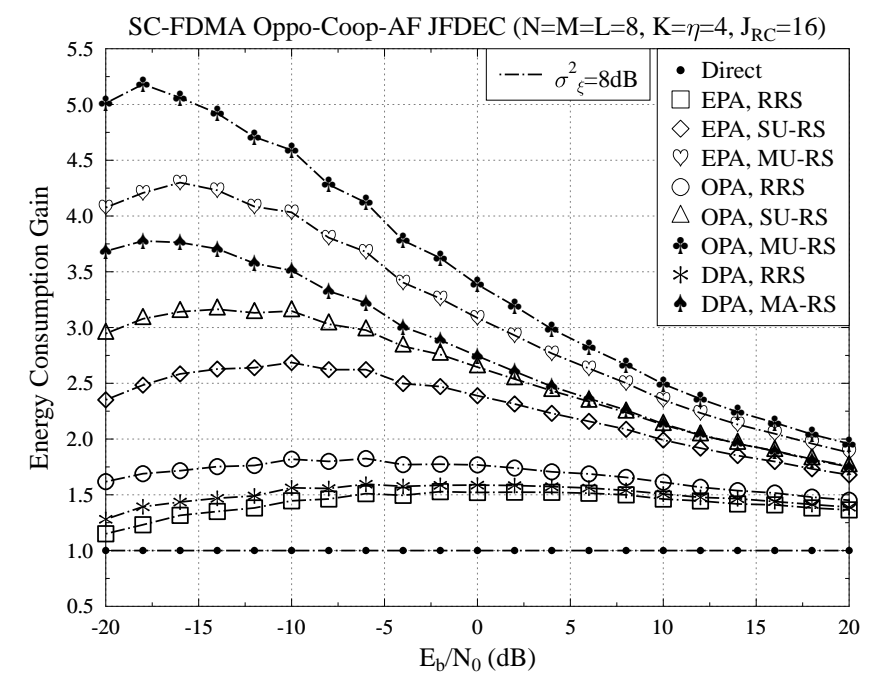

Fig. 10. Energy consumption of relay selection schemes for $8 \mathrm{~dB}$ shadowing variance

Furthermore, since we adopt the direct transmission regime in the absence of shadowing as a reference, the shadowing effects imposed on ECG are illustrated in Fig.. 11 for the MU-RS invoking EPA and in Fig. 12 for DPA aided MA-RS associated with $J=16$ under perfect power control. The ECG difference between the scenarios associated with different shadowing variances implies that opportunistic scheduling constitutes a power-efficient design, when communicating over realistic shadow fading channels associated with $\sigma_{\xi}^{2}=0,2,4,8 \mathrm{~dB}$, compared to the non-cooperative benchmarkers. Specifically, when reducing the transmit power and hence $E_{b} / N_{0}$, the ECGs of both MU-RS and MA-RS are increased more substantially for $\sigma_{\xi}^{2}=8 \mathrm{~dB}$ compared to the lower fading variances considered. For instance, the MU-RS and MA-RS may achieve an ECG of 9 and 8 at $E_{b} / N_{0}=-10 \mathrm{~dB}$, respectively.

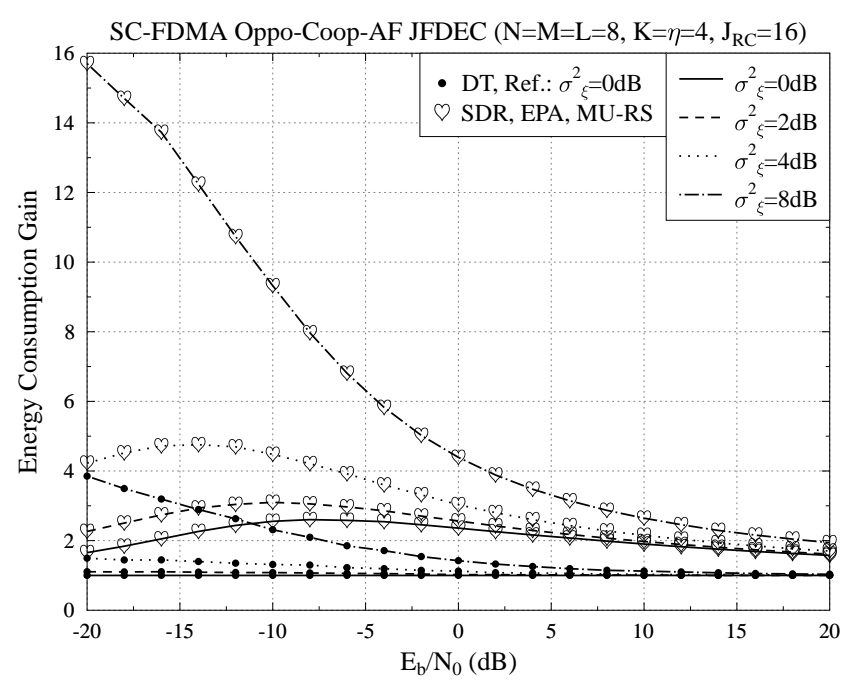

Fig. 11. Shadowing effects on energy consumption of MU-RS for SDR invoking EPA

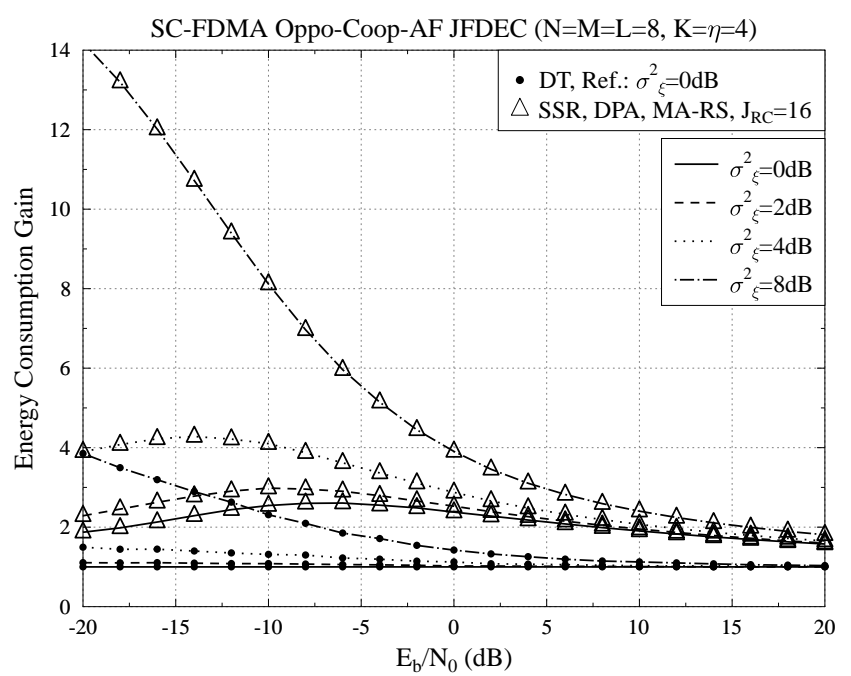

Fig. 12. Shadowing effects on energy consumption of MA-RS for SSR invoking DPA

\section{CONCLUSIONS}

In this paper we evaluated the performance benefits of the energy-efficient opportunistic AF cooperation aided multiuser SC-FDMA uplink, which was designed to be free from any MUI at the relays, when communicating over frequencyselective fading channels in shadow fading scenarios. The channel-dependent relay selection schemes were investigated considering source/relay power sharing based on the proposed JFDEC-MMSE solution, in order to exploit the multi-user selective diversity combined with cooperative diversity in the presence of both pass-loss and shadowing, while subjected 
to imperfect power control. Our results demonstrate that at a BER of $10^{-4}$, the proposed receiver is capable of saving $2 \mathrm{~dB}$ power by achieving a higher cooperative diversity gain than the conventional receiver. For instance, when the channel exhibits a shadowing variance of $8 \mathrm{~dB}$ at $E_{b} / N_{0}=-10 \mathrm{~dB}$, an ECG of $2.5 \sim 4.5$ is attainable by invoking the proposed SU-RS, MU-RS and MA-RS schemes compared to the noncooperative scenario. Most importantly, the ECG gleaned from our MU-RS and MA-RS schemes may increase to $4 \sim 8$ when the shadowing variance is increased from 4 to $8 \mathrm{~dB}$ compared to the direct transmission in the absence of shadowing at $E_{b} / N_{0}=-10 \mathrm{~dB}$.

\section{APPENDIX}

Since we have Eq. (22) in Subsection III-B, all the diagonal elements of $A_{k^{\prime}}^{\mathrm{t}}$ are equal, which are given by

$$
\begin{aligned}
a_{k^{\prime} n n}^{\mathrm{t}} & =\frac{1}{N} \operatorname{Tr}\left[\boldsymbol{A}_{k^{\prime}}^{\mathrm{t}}\right] \\
& =\frac{1}{N} \sum_{n=0}^{N-1}\left(\left(w_{k^{\prime}, n}^{\mathrm{D}}\right)^{*} h_{0, k^{\prime}, n}^{\mathrm{D}, \mathrm{f}}+\left(w_{k^{\prime},(n+N)}^{\mathrm{D}}\right)^{*} h_{1, k^{\prime}, n}^{\mathrm{D}, \mathrm{f}}\right) .
\end{aligned}
$$

The power of the desired signal at any instant is expressed by

$$
\begin{aligned}
P_{d e s} & =P_{k}^{\mathrm{S}}\left(a_{k^{\prime} n n}^{\mathrm{t}}\right)^{2} \\
& =P_{k}^{\mathrm{S}}\left[\frac{1}{N} \sum_{n=0}^{N-1}\left(\frac{\left|h_{0, k^{\prime}, n}^{\mathrm{D}, \mathrm{f}}\right|^{2}}{\sigma_{N}^{2}}+\frac{\left|h_{1, k^{\prime}, n}^{\mathrm{D}, \mathrm{f}}\right|^{2}}{\mathcal{N}_{1, n}^{\mathrm{D}}}\right) e_{k^{\prime} n}\right]^{2} .
\end{aligned}
$$

In parallel, the power of the estimated signal, which is defined as the power of the desired signal plus the power of the ISI, is given by the circulant covariance matrix of the estimated signal $\hat{\boldsymbol{y}}_{k^{\prime}}^{\mathrm{D}, \mathrm{t}}$ as follows:

$$
\begin{aligned}
P_{\text {est }} & =P_{d e s}+P_{I S I}=\frac{1}{N} \operatorname{Tr}\left\{\mathrm{E}\left[\boldsymbol{A}_{k^{\prime}}^{\mathrm{t}} \boldsymbol{x}_{k^{\prime}}^{\mathrm{t}}\left(\boldsymbol{x}_{k^{\prime}}^{\mathrm{t}}\right)^{H}\left(\boldsymbol{A}_{k^{\prime}}^{\mathrm{t}}\right)^{H}\right]\right\} \\
& =\frac{P_{k}^{\mathrm{S}}}{N} \sum_{n=0}^{N-1}\left[\left(\frac{\left|h_{0, k^{\prime}, n}^{\mathrm{D}, \mathrm{f}}\right|^{2}}{\sigma_{N}^{2}}+\frac{\left|h_{1, k^{\prime}, n}^{\mathrm{D}, \mathrm{f}}\right|^{2}}{\mathcal{N}_{1, n}^{\mathrm{D}}}\right) e_{k^{\prime} n}\right]^{2} .
\end{aligned}
$$

Additionally, the desired signal is also corrupted by the noise, whose power is given by the diagonal elements of covariance matrix of the equivalent noise at receiver, yielding

$$
\begin{aligned}
\hat{\mathcal{N}} & =\frac{1}{N} \operatorname{Tr}\left\{\mathrm{E}\left[\hat{\boldsymbol{n}}_{D}^{\mathrm{t}}\left(\hat{\boldsymbol{n}}_{D}^{\mathrm{t}}\right)^{H}\right]\right\} \\
& =\frac{1}{N} \sum_{n=0}^{N-1}\left(\frac{\left|h_{0, k^{\prime}, n}^{\mathrm{D}, \mathrm{f}}\right|^{2}}{\sigma_{N}^{2}}+\frac{\left|h_{1, k^{\prime}, n}^{\mathrm{D}, \mathrm{f}}\right|^{2}}{\mathcal{N}_{1, n}^{\mathrm{D}}}\right) e_{k^{\prime} n}^{2} .
\end{aligned}
$$

Hence, we obtain the overall signal-to-interference-and-noise ratio (SINR) $\gamma_{k^{\prime}}$ per bit simplified as below

$$
\begin{aligned}
\gamma_{k^{\prime}} & =\frac{P_{\text {des }}}{P_{I S I}+\hat{\mathcal{N}}} \\
& =\left[\left(\frac{1}{N} \sum_{n=0}^{N-1} \frac{\gamma_{k^{\prime}, n}^{\mathrm{D} 0}+\gamma_{k^{\prime}, n}^{\mathrm{D} 1}}{\gamma_{k^{\prime}, n}^{\mathrm{D} 0}+\gamma_{k^{\prime}, n}^{\mathrm{D} 1}+1}\right)^{-1}-1\right]^{-1} \\
& =\left(\frac{1}{N} \sum_{n=0}^{N-1} \frac{1}{\gamma_{k^{\prime}, n}^{\mathrm{D} 0}+\gamma_{k^{\prime}, n}^{\mathrm{D} 1}+1}\right)^{-1}-1=P_{k^{\prime}}^{\mathrm{S}} e_{k^{\prime}}^{-1}-1 .
\end{aligned}
$$

\section{REFERENCES}

[1] L. Hanzo, O. Alamri, M. ElHajjar, and N. Wu, Near-Capacity MultiFunctional MIMO Systems: Sphere-Packing, Iterative Detection and Cooperation. Wiley, 2009.

[2] A. Sendonaris, E. Erkip, and B. Aazhang, "User cooperation diversity Part I and II," IEEE Transactions on Communications, vol. 51, no. 11, pp. 1927-1948, Nov. 2003.

[3] R. U. Nabar, H. Bölcskei, and F. W. Kneubühler, "Fading relay channels: Performance limits and space-time signal design," IEEE Journal on Selected Areas in Communications, vol. 22, no. 6, pp. 1099-1109, Aug. 2004.

[4] P. Anghel and M. Kaveh, "Exact symbol error probability of a cooperative network in a Rayleigh-fading environment," IEEE Transactions on Wireless Communications, vol. 3, no. 5, pp. 1416-1421, Sep. 2004.

[5] J. N. Laneman, D. N. C. Tse, and G. W. Wornell, "Cooperative diversity in wireless networks: Efficient protocols and outage behavior," IEEE Transactions on Information Theory, vol. 50, no. 12, pp. 3062-3080, Dec. 2004.

[6] W. Fang, L.-L. Yang, and L. Hanzo, "Single-user performance of directsequence code-division multiple-access using relay diversity and power allocation," IET Proceedings on Communications, vol. 2, no. 3, pp. 462 472, Mar. 2008.

[7] R. Zhang and L. Hanzo, "Coding schemes for energy efficient multisource cooperation aided uplink transmission," IEEE Signal Processing Letters, vol. 16, no. 5, pp. 438-441, May 2009.

[8] L. Wang and L. Hanzo, "The resource-optimized differentially modulated hybrid $\mathrm{AF} / \mathrm{DF}$ cooperative cellular uplink using multiple-symbol differential sphere detection," IEEE Signal Processing Letters, vol. 16, no. 11, pp. 965-968, Nov. 2009.

[9] Y. Li, B. Vucetic, Z. Zhou, and M. Dohler, "Distributed adaptive power allocation for wireless relay networks," IEEE Transactions on Wireless Communications, vol. 6, no. 3, pp. 948-958, Mar. 2007.

[10] M. Kaneko, K. Hayashi, P. Popovski, K. Ikeda, H. Sakai, and R. Prasad, "Amplify-and-forward cooperative diversity schemes for multi-carrier systems," IEEE Transactions on Wireless Communications, vol. 7, no. 5, pp. 1845-1850, May 2008.

[11] E. C. van der Meulen, "Three-terminal communication channels," Advances in Applied Probability, vol. 3, no. 1, pp. 120-154, 1971.

[12] A. Bletsas, A. Khisti, D. Reed, and A. Lippman, "A simple cooperative diversity method based on network path selection," IEEE Journal on Selected Areas in Communications, vol. 24, no. 3, pp. 659-672, Mar. 2006.

[13] Y. Zhao, R. Adve, and T. J. Lim, "Improving amplify-and-forward relay networks: Optimal power allocation versus selection," IEEE Transactions on Wireless Communications, vol. 6, no. 8, pp. 3114-3123, Aug. 2007.

[14] A. Bletsas, H. Shin, and M. Z. Win, "Cooperative communications with outage-optimal opportunistic relaying," IEEE Transactions on Wireless Communications, vol. 6, no. 9, pp. 3450-3460, Sep. 2007.

[15] R. Madan, N. Mehta, A. Molisch, and J. Zhang, "Energy-efficient cooperative relaying over fading channels with simple relay selection," IEEE Transactions on Wireless Communications, vol. 7, no. 8, pp. 30133025, Aug. 2008.

[16] Y. Jing and H. Jafarkhani, "Single and multiple relay selection schemes and their achievable diversity orders," IEEE Transactions on Wireless Communications, vol. 8, no. 3, pp. 1414-1423, Mar. 2009.

[17] H. G. Myung, J. Lim, and D. J. Goodman, "Single carrier FDMA for uplink wireless transmission," IEEE Vehicular Technology Magazine, pp. 30-38, Sep. 2006.

[18] E-UTRA LTE Physical layer - General description, 3GPP Std. TS 35.201 (V8.3.0), 2009.

[19] D. Falconer, S. L. Ariyavisitakul, A. Benyamin-Seeyar, and B. Eidson, "Frequency domain equalization for single-carrier broadband wireless systems," IEEE Communications Magazine, pp. 58-66, Apr. 2002.

[20] N. Benvenuto, R. Dinis, D. Falconer, and S. Tomasin, "Single carrier modulation with nonlinear frequency domain equalization: An idea whose time has come-again," Proceedings of the IEEE, vol. 98, no. 1, pp. 69-96, Jan. 2010.

[21] F. Pancaldi, G. M. Vitetta, R. Kalbasi, N. Al-Dhahir, M. Uysal, and H. Mheidat, "Single-carrier frequency domain equalization," IEEE Signal Processing Magazine, pp. 37-56, Sep. 2008.

[22] L. Hanzo, M. Münster, B.-J. Choi, and T. Keller, OFDM and MC-CDMA for Broadband Multi-User Communications, WLANs and Broadcasting. Wiley, 2003.

[23] H. G. Myung and D. J. Goodman, Single Carrier FDMA: A New Air Interface for Long Term Evolution. Wiley, 2008. 
[24] L.-L. Yang, Multicarrier Communications. Wiley, 2009.

[25] J. Zhang, L.-L. Yang, and L. Hanzo, "Multi-user performance of the amplify-and-forward single-relay assisted SC-FDMA uplink," in Proceedings of the 2009 IEEE 70th Vehicular Technology Conference Fall (VTC 2009-Fall), Sep. 2009, pp. 1-5.

[26] H. Gacanin and F. Adachi, "A performance of cooperative relay network based on OFDM/TDM using MMSE-FDE in a wireless channel," in Proceedings of the 2009 IEEE 70th Vehicular Technology Conference Fall (VTC 2009-Fall), Sep. 2009, pp. 1-5.

[27] A. Goldsmith, Wireless Communications. Cambridge University Press, 2005.

[28] G. Kadel, "Diversity and equalization in frequency domain a robust and flexible receiver technology for broadband mobile communication systems," in Proceedings of IEEE Vehicular Technology Conference, vol. 2, May 1997, pp. 894-898.

[29] M. V. Clark, "Adaptive frequency-domain equalization and diversity combining for broadband wireless communications," IEEE Journal on Selected Areas in Communications, vol. 16, no. 8, pp. 1385-1395, Oct. 1998.

[30] A. Gusmao, R. Dinis, and N. Esteves, "On frequency-domain equalization and diversity combining for broadband wireless communications," IEEE Transactions on Communications, vol. 51, no. 7, pp. 1029-1033, Jul. 2003.

[31] J. Coon, S. Armour, M. Beach, and J. McGeehan, "Adaptive frequencydomain equalization for single-carrier multiple-input multiple-output wireless transmissions," IEEE Transactions on Signal Processing, vol. 53, no. 8, pp. 3247-3256, Aug. 2005.

[32] J.-S. Baek and J.-S. Seo, "Efficient design of block adaptive equalization and diversity combining for space-time block-coded single-carrier systems," IEEE Transactions on Wireless Communications, vol. 7, no. 7, pp. 2608-2611, Jul. 2008

[33] H. Xiong, J. Xu, and P. Wang, "Frequency-domain equalization and diversity combining for demodulate-and-forward cooperative systems," in Proceedings of the IEEE International Conference on Acoustics, Speech and Signal Processing, 2008 (ICASSP 2008), Mar. 2008, pp. $3245-3248$.

[34] K. S. Woo, Y. J. Kim, H. I. Yoo, J. Kim, S. Yun, and Y. S. Cho, "An improved receive diversity combining technique for SC-FDMA-based cooperative relays," in Proceedings of the 2009 IEEE 70th Vehicular Technology Conference Fall (VTC 2009-Fall), Sep. 2009, pp. 1 -5.

[35] K. S. Woo, H. Yim, Y. J. Kim, H. I. Yoo, and Y. S. Cho, "An efficient receive-diversity-combining technique for sc-fdma-based cooperative relays," IEEE Transactions on Vehicular Technology, vol. 59, no. 8, pp. 4187-4191, Oct. 2010.

[36] N. Kong and L. Milstein, "Error probability of multicell CDMA over frequency selective fading channels with power control error," IEEE Transactions on Communications, vol. 47, no. 4, pp. 608-617, Apr 1999.

[37] Z. Han, T. Himsoon, W. Siriwongpairat, and K. Liu, "Resource allocation for multiuser cooperative OFDM networks: Who helps whom and how to cooperate," IEEE Transactions on Vehicular Technology, vol. 58 , no. 5, pp. $2378-2391$, Jun. 2009.

[38] Y. Ding and M. Uysal, "Amplify-and-forward cooperative OFDM with multiple-relays: performance analysis and relay selection methods," IEEE Transactions on Wireless Communications, vol. 8, no. 10, pp. 4963 -4968 , Oct. 2009.

[39] K. Vardhe, D. Reynolds, and B. Woerner, "Joint power allocation and relay selection for multiuser cooperative communication," IEEE Transactions on Wireless Communications, vol. 9, no. 4, pp. 1255 1260, Apr. 2010

[40] J. Zhang, L.-L. Yang, and L. Hanzo, "Power-efficient opportunistic amplify-and-forward single-relay aided multi-user SC-FDMA uplink," in Proceedings of the IEEE Vehicular Technology Conference 2010 Spring, May 2010, pp. 1-5.

[41] C. Han, T. Harrold, I. Krikidis, I. Ku, and et.al, "Green radio: Radio techniques to enable energy efficient wireless networks," IEEE Communications Magazine, pp. 1-8, 2010, accepted for publication.

[42] T. S. Rappaport, Wireless Communications, 2nd Edition. Prentice Hall PTR, 2002.

[43] D. Z. Filho, L. Féty, and M. Terré, "A hybird single-carrier/multicarrie transmission scheme with power allocation," EURASIP Journal on Wireless Communications and Networking, vol. 2008, pp. 1-11, 2008.

[44] U. Madhow and M. L. Honig, "MMSE interference suppression for direct-sequence spread-spectrum CDMA," IEEE Transactions on Communications, vol. 42, pp. 3178-3188, Dec. 1994.

[45] J. G. Proakis, Digital Communications, 4th Edition. McGraw Hill, 2000.

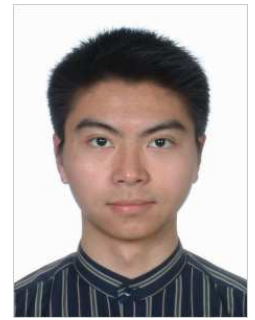

Jiayi Zhang (S'07) received his B.Eng. degree from Nanjing University of Aeronautics and Astronautics (NUAA), China, and the M.Sc. degree with distinction from University of Southampton, UK, in 2006 and 2007, respectively. He is currently continuing his study toward the Ph.D. degree within the Communications Research Group, School of Electronics and Computer Science at University of Southampton, UK and he is also involved in the Mobile VCE's Core 4 Delivery Efficiency and Core 5 Green Radio projects. His research interests in wireless communications include energy-efficient cooperative relaying, multicell cooperation and resource allocation for SC-FDMA, MIMO-OFDM as well as beyond 3GPP LTE-Advanced systems.

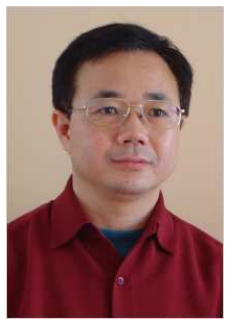

Lie-Liang Yang (M'98-SM'02) received the B.Eng. degree in communications engineering from Shanghai Tiedao University, Shanghai, China, in 1988 and the M.Eng. and Ph.D. degrees in communications and electronics from Northern (Beijing) Jiaotong University, Beijing, China, in 1991 and 1997, respectively. From June 1997 to December 1997, he was a Visiting Scientist with the Institute of Radio Engineering and Electronics, Academy of Sciences of the Czech Republic, Prague, Czech Republic. Since December 1997, he has been with the University of Southampton, Southampton, U.K., where he is currently a Professor with the School of Electronics and Computer Science. He has published more than 240 research papers in journals and conference proceedings, authored/coauthored three books, and published several book chapters. Details about his publications can be found at http://wwwmobile.ecs.soton.ac.uk/lly/. His research has covered a wide range of topics in wireless communications, networking, and signal processing.

Dr. Yang is currently an Associate Editor of the IEEE Transactions on Vehicular Technology, the Journal of Communications and Networks, and the Security and Communication Networks journal.

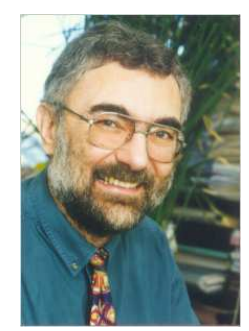

Lajos Hanzo FREng, FIEEE, FIET, DSc received his degree in electronics in 1976 and his doctorate in 1983. In 2009 he was awarded the honorary doctorate "Doctor Honaris Causa". During his 34-year career in telecommunications he has held various research and academic posts in Hungary, Germany and the UK. Since 1986 he has been with the School of Electronics and Computer Science, University of Southampton, UK, where he holds the chair in telecommunications. He has co-authored 20 John Wiley - IEEE Press books on mobile radio communications totalling in excess of 10000 pages, published about 970 research entries at IEEE Xplore, acted as TPC Chair of IEEE conferences, presented keynote lectures and been awarded a number of distinctions. Currently he is directing an academic research team, working on a range of research projects in the field of wireless multimedia communications sponsored by industry, the Engineering and Physical Sciences Research Council (EPSRC) UK, the European IST Programme and the Mobile Virtual Centre of Excellence (VCE), $\mathrm{UK}$. He is an enthusiastic supporter of industrial and academic liaison and he offers a range of industrial courses. He is also a Governor of both the IEEE ComSoc and the VTS. He is the Editor-in-Chief of the IEEE Press and a Chaired Prof. also at Tsinghua University, Beijing. For further information on research in progress and associated publications please refer to http://www-mobile.ecs.soton.ac.uk. 